\title{
The Gauss image of entire graphs of higher codimension and Bernstein type theorems
}

\author{
J. Jost · Y. L. Xin · Ling Yang
}

Received: 19 September 2010 / Accepted: 20 July 2011 / Published online: 6 June 2012

(C) The Author(s) 2012. This article is published with open access at Springerlink.com

\begin{abstract}
Under suitable conditions on the range of the Gauss map of a complete submanifold of Euclidean space with parallel mean curvature, we construct a strongly subharmonic function and derive a-priori estimates for the harmonic Gauss map. The required conditions here are more general than in previous work and they therefore enable us to improve substantially previous results for the Lawson-Osseman problem concerning the regularity of minimal submanifolds in higher codimension and to derive Bernstein type results.
\end{abstract}

\section{Mathematics Subject Classification (1991) $\quad 58 \mathrm{E} 20 \cdot 53 \mathrm{~A} 10$}

\section{Introduction}

We consider an oriented $n$-dimensional submanifold $M$ in $\mathbb{R}^{n+m}$ with $n \geq 3, m \geq 2$. The Gauss map $\gamma: M \rightarrow \mathbf{G}_{n, m}$ maps $M$ into a Grassmann manifold. In fact, for codimension $m=1$, this Grassmann manifold $\mathbf{G}_{n, 1}$ is the unit sphere $S^{n}$. In this paper, however, we are interested in the case $m \geq 2$ where the geometry of this Grassmann manifold is more complicated. By the theorem of Ruh and Vilms [16], $\gamma$ is harmonic if and only if $M$ has parallel mean curvature. This result applies thus in particular to the case where $M$ is a minimal submanifold of Euclidean space.

Communicated by L. Ambrosio.

J. Jost $(\varangle) \cdot$ L. Yang

Max Planck Institute for Mathematics in the Sciences, Inselstr. 22, 04103 Leipzig, Germany

e-mail: jost@mis.mpg.de

Y. L. Xin

Institute of Mathematics, Fudan University, Shanghai 200433, China

e-mail: ylxin@fudan.edu.cn

Present Address:

L. Yang

Institute of Mathematics, Fudan University, Shanghai 200433, China

e-mail: yanglingfd@fudan.edu.cn 
Now, the Bernstein problem for entire minimal graphs is one of the central problems in geometric analysis. Let us summarize the status of this problem, first for the case of codimension 1. The central result is that an entire minimal graph $M$ of dimension $n \leq 7$ and codimension 1 has to be planar, but there are counterexamples to such a Bernstein type theorem in dimension 8 or higher. However, when the additional condition is imposed that the slope of the graph be uniformly bounded, then a theorem of Moser [15], called a weak Bernstein theorem asserts that such an $M$ in arbitrary dimension has to be planar. Thus, the counterexamples arise from a non-uniform behavior at infinity. In fact, by a general scaling argument, the Bernstein theorems are intimately related to the regularity question for the minimal hypersurface equation.

A natural and important question then is to what extent such Bernstein type theorems generalize to entire minimal graphs of codimension $m \geq 2$. Moser's result has been extended to higher codimension by Chern and Osserman [3] for dimension $n=2$ and Barbosa [2] and Fisher and Colbrie [5] for dimension $n=3$. For dimension $n=4$ and codimension $m=3$, however, there is a counterexample given by Lawson and Osserman [14]. In fact, their paper emphasizes the stark contrast between the cases of codimension 1 and greater than 1 for the minimal submanifold system, concerning regularity, uniqueness, and existence. The Lawson-Osserman problem then is concerned with a systematic understanding of the analytic aspects of the minimal submanifold system in higher codimension. As in the case of codimension 1, the Bernstein problem provides a key towards this aim.

While the work of Lawson-Osserman produced a counterexample for a general Bernstein theorem, there are also some positive results in this direction which we shall now summarize. Hildebrandt et al. [10] started a systematic approach on the basis of the aforementioned Ruh-Vilms theorem. That is, they developed and employed the theory of harmonic maps and the convex geometry of Grassmannian manifolds, and obtained Bernstein type results in general dimension and codimension. Their main result says that a Bernstein result holds if the image of the Gauss map is contained in a strictly convex distance ball. Since the Riemannian sectional curvature of $\mathbf{G}_{n, m}$ is nonnegative, the maximal radius of such a convex ball is bounded. In codimension 1, this in particular reproduces Moser's theorem, and in this sense, their result is optimal. For higher codimension, their result can be improved, for the following reason. Since the sectional curvature of $\mathbf{G}_{n, m}$ for $n, m \geq 2$ is not constant, there exist larger convex sets than geodesic distance balls, and it turns out that harmonic (e.g. Gauss) maps with values in such convex sets can still be well enough controlled. In this sense, the results of [10] could be improved by Jost and Xin [12], Wang [18] and Xin and Yang [21]. In [12], the largest such geodesically convex set in a Grassmannian manifold was found. Formulating it somewhat differently, the harmonic map approach is based on the fact that the composition of a harmonic map with a convex function is a subharmonic function, and by using quantitative estimates for such subharmonic functions, regularity and Liouville type results for harmonic maps can be obtained. Thus, convex functions were systematically utilized in [21]. In this paper, also the fundamental connection between estimates for the second fundamental form of minimal submanifolds and estimates for their Gauss maps was systematically explored. On this basis, the fundamental curvature estimate technique, as developed by Schoen et al. [17] and Ecker and Huisken [4], could be used in [21].

Still, there remains a large quantitative gap between those positive results and the counterexample of Lawson-Osserman. In this situation, it could either be that Bernstein theorems can be found under more general conditions, or that there exist other counterexamples in the so far unexplored range. 
In the present paper, we make a step towards closing this gap in the positive direction. We identify a geometrically natural function $v$ on a Grassmann manifold and a natural quantitative condition under which the precomposition of this function with a harmonic (Gauss) map is (strongly) subharmonic (Theorem 3.1). When the precomposition of $v$ with the Gauss map of a complete minimal submanifold is bounded, then that submanifold is an entire graph of bounded slope. On one hand, this is the first systematic example in harmonic map regularity theory where this auxiliary function is not necessarily convex. On the other hand, the Lawson-Osserman's counterexample can also be readily characterized in terms of this function. Still, the range of values for $v$ where we can apply our scheme is strictly separated from the value of $v$ in that example. Therefore, still some gap remains which should be explored in future work.

Our work also finds its natural position in the general regularity theory for harmonic maps. Also, once we have a strongly subharmonic function, we could derive Bernstein type results within the frame work of geometric measure theory, by the standard blow-down procedure and appeal to Allard's regularity theorem [1]. By building upon the work of many people on harmonic map regularity, we can obtain more insight, however. In particular, we shall use the iteration method of [10], we can explore the relation with curvature estimates, and we shall utilize a version of the telescoping trick (Theorem 4.1) to finally obtain a quantitatively controlled Gauss image shrinking process (Theorems 5.1 and 6.1). In this way, we can understand why the submanifold is flat as the Bernstein result asserts. More precisely, we obtain the following Bernstein type result, which substantially improves our previous results.

Theorem 1.1 Let $z^{\alpha}=f^{\alpha}\left(x^{1}, \ldots, x^{n}\right), \alpha=1, \ldots, m$, be smooth functions defined everywhere in $\mathbb{R}^{n}(n \geq 3, m \geq 2)$. Suppose their graph $M=(x, f(x))$ is a submanifold with parallel mean curvature in $\mathbb{R}^{n+m}$. Suppose that there exists a number $\beta_{0}<3$ such that

$$
\Delta_{f}=\left[\operatorname{det}\left(\delta_{i j}+\sum_{\alpha} \frac{\partial f^{\alpha}}{\partial x^{i}} \frac{\partial f^{\alpha}}{\partial x^{j}}\right)\right]^{\frac{1}{2}} \leq \beta_{0} .
$$

Then $f^{1}, \ldots, f^{m}$ has to be affine linear, i.e., it represents an affine $n$-plane.

The essential point is to show that $v:=\Delta_{f}$ is subharmonic when $<3$. In fact, when $v \leq \beta_{0}<3$, then $\Delta v \geq K_{0}|B|^{2}$ where $K_{0}$ is a positive constant and $B$ is the second fundamental form of $M$ in $\mathbb{R}^{n+m}$. This principle is not new. Wang [18] has given conditions under which $\log v$ is subharmonic and has derived Bernstein results from this, as indicated above. He only needs that $v$ be uniformly bounded by some constant, not necessarily $<3$, but in addition that there exist some $\delta>0$ such that for any two eigenvalues $\lambda_{i}, \lambda_{j}$ with $i \neq j$, the inequality $\left|\lambda_{i} \lambda_{j}\right| \leq 1-\delta$ holds (the latter condition means in geometric terms that $d f$ is strictly area decreasing on any two-dimensional subspace). Since subharmonicity of $\log v$ is a weaker property than subharmonicity of $v$ itself, his computation is substantially easier than ours, and our results cannot be deduced from his. In fact, $v^{2}=\prod\left(1+\lambda_{i}^{2}\right)$, and while the condition of [12] which can be reformulated as $v^{2}$ being bounded away from 4 implies the condition of [18] so that the latter result generalizes the former, the condition needed in the present paper is only the weaker one that $v^{2}$ be bounded away from 9 .

In fact, somewhat more refined results can be obtained, as will be pointed out in the final remarks of this paper. 


\section{Geometry of Grassmann manifolds}

Let $\mathbb{R}^{n+m}$ be an $(n+m)$-dimensional Euclidean space. Its oriented $n$-subspaces constitute the Grassmann manifold $\mathbf{G}_{n, m}$, which is the Riemannian symmetric space of compact type $S O(n+m) / S O(n) \times S O(m)$.

$\mathbf{G}_{n, m}$ can be viewed as a submanifold of some Euclidean space via the Plücker embedding. The restriction of the Euclidean inner product on $M$ is denoted by $w: \mathbf{G}_{n, m} \times \mathbf{G}_{n, m} \rightarrow \mathbb{R}$

$$
w(P, Q)=\left\langle e_{1} \wedge \cdots \wedge e_{n}, f_{1} \wedge \cdots \wedge f_{n}\right\rangle=\operatorname{det} W
$$

where $P$ is spanned by a unit $n$-vector $e_{1} \wedge \cdots \wedge e_{n}, Q$ is spanned by another unit $n$-vector $f_{1} \wedge \cdots \wedge f_{n}$, and $W=\left(\left\langle e_{i}, f_{j}\right\rangle\right)$. It is well-known that

$$
W^{T} W=O^{T} \Lambda O
$$

with $O$ an orthogonal matrix and

$$
\Lambda=\left(\begin{array}{lll}
\mu_{1}^{2} & & \\
& \ddots & \\
& & \mu_{n}^{2}
\end{array}\right) .
$$

Here each $0 \leq \mu_{i}^{2} \leq 1$. Putting $p:=\min \{m, n\}$, then at most $p$ elements in $\left\{\mu_{1}^{2}, \ldots, \mu_{n}^{2}\right\}$ are not equal to 0 . Without loss of generality, we can assume $\mu_{i}^{2}=1$ whenever $i>p$. We also note that the $\mu_{i}^{2}$ can be expressed as

$$
\mu_{i}^{2}=\frac{1}{1+\lambda_{i}^{2}} .
$$

The Jordan angles between $P$ and $Q$ are defined by

$$
\theta_{i}=\arccos \left(\mu_{i}\right) \quad 1 \leq i \leq p .
$$

The distance between $P$ and $Q$ is defined by

$$
d(P, Q)=\sqrt{\sum \theta_{i}^{2}} .
$$

Thus, (2.1) becomes

$$
\lambda_{i}=\tan \theta_{i}
$$

In the sequel, we shall assume $n \geq m$ without loss of generality. We use the summation convention and agree on the ranges of indices:

$$
1 \leq i, j, k, l \leq n, \quad 1 \leq \alpha, \beta, \gamma \leq m, \quad a, b, \ldots=1, \ldots, n+m .
$$

Now we fix $P_{0} \in \mathbf{G}_{n, m}$. We represent it by $n$ vectors $\epsilon_{i}$, which are complemented by $m$ vectors $\epsilon_{n+\alpha}$, such that $\left\{\epsilon_{i}, \epsilon_{n+\alpha}\right\}$ form an orthonormal base of $\mathbb{R}^{m+n}$.

Denote

$$
\mathbb{U}:=\left\{P \in \mathbf{G}_{n, m}, w\left(P, P_{0}\right)>0\right\} .
$$

We can span an arbitrary $P \in \mathbb{U}$ by $n$-vectors $f_{i}$ :

$$
f_{i}=\epsilon_{i}+Z_{i \alpha} \epsilon_{n+\alpha} .
$$


The canonical metric in $\mathbb{U}$ can be described as

$$
d s^{2}=\operatorname{tr}\left(\left(I_{n}+Z Z^{T}\right)^{-1} d Z\left(I_{m}+Z^{T} Z\right)^{-1} d Z^{T}\right),
$$

where $Z=\left(Z_{i \alpha}\right)$ is an $(n \times m)$-matrix and $I_{n}$ (res. $\left.I_{m}\right)$ denotes the $(n \times n)$-identity (res. $m \times m$ ) matrix. It is shown that (2.4) can be derived from (2.2) in [20].

For any $P \in \mathbb{U}$, the Jordan angles between $P$ and $P_{0}$ are defined by $\left\{\theta_{i}\right\}$. Let $E_{i \alpha}$ be the matrix with 1 in the intersection of row $i$ and column $\alpha$ and 0 otherwise. Then, $\sec \theta_{i} \sec \theta_{\alpha} E_{i \alpha}$ form an orthonormal basis of $T_{P} \mathbf{G}_{n, m}$ with respect to (2.4). Denote its dual frame by $\omega_{i \alpha}$.

Our fundamental quantity will be

$$
v\left(\cdot, P_{0}\right):=w^{-1}\left(\cdot, P_{0}\right) \text { on } \mathbb{U} .
$$

For arbitrary $P \in \mathbb{U}$ determined by an $n \times m$ matrix $Z$, it is easily seen that

$$
v\left(P, P_{0}\right)=\left[\operatorname{det}\left(I_{n}+Z Z^{T}\right)\right]^{\frac{1}{2}}=\prod_{\alpha=1}^{m} \sec \theta_{\alpha}=\prod_{\alpha=1}^{m} \frac{1}{\mu_{\alpha}} .
$$

where $\theta_{1}, \ldots, \theta_{m}$ denote the Jordan angles between $P$ and $P_{0}$.

In this terminology, $\operatorname{Hess}\left(v\left(\cdot, P_{0}\right)\right)$ has been estimated in [21]. By (3.8) in [21], we have

$$
\begin{aligned}
\operatorname{Hess}\left(v\left(\cdot, P_{0}\right)\right)= & \sum_{i \neq \alpha} v \omega_{i \alpha}^{2}+\sum_{\alpha}\left(1+2 \lambda_{\alpha}^{2}\right) v \omega_{\alpha \alpha}^{2}+\sum_{\alpha \neq \beta} \lambda_{\alpha} \lambda_{\beta} v\left(\omega_{\alpha \alpha} \otimes \omega_{\beta \beta}+\omega_{\alpha \beta} \otimes \omega_{\beta \alpha}\right) \\
= & \sum_{m+1 \leq i \leq n, \alpha} v \omega_{i \alpha}^{2}+\sum_{\alpha}\left(1+2 \lambda_{\alpha}^{2}\right) v \omega_{\alpha \alpha}^{2}+\sum_{\alpha \neq \beta} \lambda_{\alpha} \lambda_{\beta} v \omega_{\alpha \alpha} \otimes \omega_{\beta \beta} \\
& +\sum_{\alpha<\beta}\left[\left(1+\lambda_{\alpha} \lambda_{\beta}\right) v\left(\frac{\sqrt{2}}{2}\left(\omega_{\alpha \beta}+\omega_{\beta \alpha}\right)\right)^{2}\right. \\
& \left.+\left(1-\lambda_{\alpha} \lambda_{\beta}\right) v\left(\frac{\sqrt{2}}{2}\left(\omega_{\alpha \beta}-\omega_{\beta \alpha}\right)\right)^{2}\right]
\end{aligned}
$$

It follows that

$$
v\left(\cdot, P_{0}\right)^{-1} \operatorname{Hess}\left(v\left(\cdot, P_{0}\right)\right)=g+\sum_{\alpha} 2 \lambda_{\alpha}^{2} \omega_{\alpha \alpha}^{2}+\sum_{\alpha \neq \beta} \lambda_{\alpha} \lambda_{\beta}\left(\omega_{\alpha \alpha} \otimes \omega_{\beta \beta}+\omega_{\alpha \beta} \otimes \omega_{\beta \alpha}\right) .
$$

The canonical Riemannian metric on $\mathbf{G}_{n, m}$ derived from (2.2) can also be described by the moving frame method. This will be useful for understanding some of the sequel. Let $\left\{e_{i}, e_{n+\alpha}\right\}$ be a local orthonormal frame field in $\mathbb{R}^{n+m}$. Let $\left\{\omega_{i}, \omega_{n+\alpha}\right\}$ be its dual frame field so that the Euclidean metric is

$$
g=\sum_{i} \omega_{i}^{2}+\sum_{\alpha} \omega_{n+\alpha}^{2}
$$

The Levi-Civita connection forms $\omega_{a b}$ of $\mathbb{R}^{n+m}$ are uniquely determined by the equations

$$
\begin{aligned}
& d \omega_{a}=\omega_{a b} \wedge \omega_{b}, \\
& \omega_{a b}+\omega_{b a}=0 .
\end{aligned}
$$


It is shown in [20] that the canonical Riemannian metric on $\mathbf{G}_{n, m}$ can be written as

$$
d s^{2}=\sum_{i, \alpha} \omega_{i n+\alpha}^{2} .
$$

\section{Subharmonic functions}

Let $M^{m} \rightarrow \mathbb{R}^{n+m}$ be an isometric immersion with second fundamental form $B$. Around any point $p \in M$, we choose an orthonormal frame field $e_{i}, \ldots, e_{n+m}$ in $\mathbb{R}^{n+m}$, such that $\left\{e_{i}\right\}$ are tangent to $M$ and $\left\{e_{n+\alpha}\right\}$ normal to $M$. The metric on $M$ is $g=\sum_{i} \omega_{i}^{2}$. We have the structure equations

$$
\omega_{i n+\alpha}=h_{\alpha i j} \omega_{j},
$$

where $h_{\alpha i j}$ are the coefficients of second fundamental form $B$ of $M$ in $\mathbb{R}^{n+m}$.

Let 0 be the origin of $\mathbb{R}^{n+m}, S O(m+n)$ be the Lie group consisting of all orthonormal frames $\left(0 ; e_{i}, e_{n+\alpha}\right), T F=\left\{\left(p ; e_{1}, \ldots, e_{n}\right): p \in M, e_{i} \in T_{p} M,\left\langle e_{i}, e_{j}\right\rangle=\delta_{i j}\right\}$ be the principle bundle of orthonormal tangent frames over $M$, and $N F=\left\{\left(p ; e_{n+1}, \ldots, e_{n+m}\right)\right.$ : $\left.p \in M, e_{n+\alpha} \in N_{p} M\right\}$ be the principle bundle of orthonormal normal frames over $M$. Then $\bar{\pi}: T F \oplus N F \rightarrow M$ is the projection with fiber $S O(n) \times S O(m)$.

The Gauss map $\gamma: M \rightarrow \mathbf{G}_{n, m}$ is defined by

$$
\gamma(p)=T_{p} M \in \mathbf{G}_{n, m}
$$

via the parallel translation in $\mathbb{R}^{n+m}$ for every $p \in M$. Then the following diagram commutes

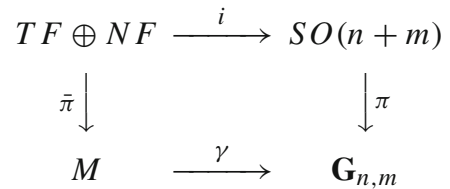

where $i$ denotes the inclusion map and $\pi: S O(n+m) \rightarrow \mathbf{G}_{n, m}$ is defined by

$$
\left(0 ; e_{i}, e_{n+\alpha}\right) \mapsto e_{1} \wedge \cdots \wedge e_{n} .
$$

It follows that

$$
|d \gamma|^{2}=\sum_{\alpha, i, j} h_{\alpha i j}^{2}=|B|^{2} .
$$

(2.8) was computed for the metric (2.4) whose corresponding coframe field is $\omega_{i \alpha}$. Since (2.4) and (2.9) are equivalent to each other, at any fixed point $P \in \mathbf{G}_{n, m}$ there exists an isotropic group action, i.e., an $S O(n) \times S O(m)$ action, such that $\omega_{i \alpha}$ is transformed to $\omega_{i} n+\alpha$, namely, there are a local tangent frame field and a local normal frame field such that at the point under consideration,

$$
\omega_{i n+\alpha}=\gamma^{*} \omega_{i \alpha} .
$$

In conjunction with (3.1) and (3.3) we obtain

$$
\gamma^{*} \omega_{i \alpha}=h_{\alpha i j} \omega_{j} .
$$

By the Ruh-Vilms theorem [16], the mean curvature of $M$ is parallel if and only if its Gauss map is a harmonic map. Now, we assume that $M$ has parallel mean curvature. 
We define

$$
v:=v\left(\cdot, P_{0}\right) \circ \gamma,
$$

This function $v$ on $M$ will be the source of the basic inequality for this paper. Its geometric significance is seen from the following observation. If the $v$-function has an upper bound (or the $w$-function has a positive lower bound), $M$ can be described as an entire graph on $\mathbb{R}^{n}$ by $f: \mathbb{R}^{n} \rightarrow \mathbb{R}^{m}$, provided $M$ is complete. In this situation, $\lambda_{i}$ is the singular values of $d f$ and

$$
v=\left[\operatorname{det}\left(\delta_{i j}+\sum_{\alpha} \frac{\partial f^{\alpha}}{\partial x^{i}} \frac{\partial f^{\alpha}}{\partial x^{j}}\right)\right]^{\frac{1}{2}}
$$

Using the composition formula, in conjunction with (2.8), (3.2) and (3.4), and the fact that $\tau(\gamma)=0$ (the tension field of the Gauss map vanishes [16]), we deduce the important formula of Lemma 1.1 in [5] and Prop. 2.1 in [18].

Proposition 3.1 Let $M$ be an $n$-submanifold in $\mathbb{R}^{n+m}$ with parallel mean curvature. Then

$$
\Delta v=v|B|^{2}+v \sum_{\alpha, j} 2 \lambda_{\alpha}^{2} h_{\alpha, \alpha j}^{2}+v \sum_{\alpha \neq \beta, j} \lambda_{\alpha} \lambda_{\beta}\left(h_{\alpha, \alpha j} h_{\beta, \beta j}+h_{\alpha, \beta j} h_{\beta, \alpha j}\right),
$$

where $h_{\alpha, i j}$ are the coefficients of the second fundamental form of $M$ in $\mathbb{R}^{n+m}$ (see 3.1).

A crucial step in this paper is to find a condition which guarantees the strong subharmonicity of the $v$-function on $M$. More precisely, under a condition on $v$, we shall bound its Laplacian from below by a positive constant times squared norm of the second fundamental form.

Looking at the expression (3.7), we group its terms according to the different types of the indices of the coefficients of the second fundamental form as follows.

$$
v^{-1} \Delta v=\sum_{\alpha} \sum_{i, j>m} h_{\alpha, i j}^{2}+\sum_{j>m} I_{j}+\sum_{j>m, \alpha<\beta} I I_{j \alpha \beta}+\sum_{\alpha<\beta<\gamma} I I I_{\alpha \beta \gamma}+\sum_{\alpha} I V_{\alpha}
$$

where

$$
\begin{gathered}
I_{j}=\sum_{\alpha}\left(2+2 \lambda_{\alpha}^{2}\right) h_{\alpha, \alpha j}^{2}+\sum_{\alpha \neq \beta} \lambda_{\alpha} \lambda_{\beta} h_{\alpha, \alpha j} h_{\beta, \beta j}, \\
I I_{j \alpha \beta}=2 h_{\alpha, \beta j}^{2}+2 h_{\beta, \alpha j}^{2}+2 \lambda_{\alpha} \lambda_{\beta} h_{\alpha, \beta j} h_{\beta, \alpha j}, \\
I I I_{\alpha \beta \gamma}=2 h_{\alpha, \beta \gamma}^{2}+2 h_{\beta, \gamma \alpha}^{2}+2 h_{\gamma, \alpha \beta}^{2}+2 \lambda_{\alpha} \lambda_{\beta} h_{\alpha, \beta \gamma} h_{\beta, \gamma \alpha} \\
+2 \lambda_{\beta} \lambda_{\gamma} h_{\beta, \gamma \alpha} h_{\gamma, \alpha \beta}+2 \lambda_{\gamma} \lambda_{\alpha} h_{\gamma, \alpha \beta} h_{\alpha, \beta \gamma}
\end{gathered}
$$

and

$$
\begin{gathered}
I V_{\alpha}=\left(1+2 \lambda_{\alpha}^{2}\right) h_{\alpha, \alpha \alpha}^{2}+\sum_{\beta \neq \alpha}\left(h_{\alpha, \beta \beta}^{2}+\left(2+2 \lambda_{\beta}^{2}\right) h_{\beta, \beta \alpha}^{2}\right) \\
+\sum_{\beta \neq \gamma} \lambda_{\beta} \lambda_{\gamma} h_{\beta, \beta \alpha} h_{\gamma, \gamma \alpha}+2 \sum_{\beta \neq \alpha} \lambda_{\alpha} \lambda_{\beta} h_{\alpha, \beta \beta} h_{\beta, \beta \alpha} .
\end{gathered}
$$

It is easily seen that

$$
I_{j}=\left(\sum_{\alpha} \lambda_{\alpha} h_{\alpha, \alpha j}\right)^{2}+\sum_{\alpha}\left(2+\lambda_{\alpha}^{2}\right) h_{\alpha, \alpha j}^{2} \geq 2 \sum_{\alpha} h_{\alpha, \alpha j}^{2} .
$$


Obviously

$$
I I_{j \alpha \beta}=\lambda_{\alpha} \lambda_{\beta}\left(h_{\alpha, \beta j}+h_{\beta, \alpha j}\right)^{2}+\left(2-\lambda_{\alpha} \lambda_{\beta}\right)\left(h_{\alpha, \beta j}^{2}+h_{\beta, \alpha j}^{2}\right) .
$$

$v=\left(\prod_{\alpha}\left(1+\lambda_{\alpha}^{2}\right)\right)^{\frac{1}{2}}$ implies $\left(1+\lambda_{\alpha}^{2}\right)\left(1+\lambda_{\beta}^{2}\right) \leq v^{2}$. Assume $\left(1+\lambda_{\alpha}^{2}\right)\left(1+\lambda_{\beta}^{2}\right) \equiv C \leq v^{2}$, then differentiating both sides implies

$$
\frac{\lambda_{\alpha} d \lambda_{\alpha}}{1+\lambda_{\alpha}^{2}}+\frac{\lambda_{\beta} d \lambda_{\beta}}{1+\lambda_{\beta}^{2}}=0
$$

Therefore

$$
\begin{aligned}
d\left(\lambda_{\alpha} \lambda_{\beta}\right) & =\lambda_{\beta} d \lambda_{\alpha}+\lambda_{\alpha} d \lambda_{\beta} \\
& =\left[\lambda_{\beta}^{2}\left(1+\lambda_{\alpha}^{2}\right)-\lambda_{\alpha}^{2}\left(1+\lambda_{\beta}^{2}\right)\right] \frac{d \lambda_{\alpha}}{\lambda_{\beta}\left(1+\lambda_{\alpha}^{2}\right)} \\
& =\left(\lambda_{\beta}^{2}-\lambda_{\alpha}^{2}\right) \frac{d \lambda_{\alpha}}{\lambda_{\beta}\left(1+\lambda_{\alpha}^{2}\right)} .
\end{aligned}
$$

It follows that $\left(\lambda_{\alpha}, \lambda_{\beta}\right) \mapsto \lambda_{\alpha} \lambda_{\beta}$ attains its maximum at the point satisfying $\lambda_{\alpha}=\lambda_{\beta}$, which is hence $\left(\left(C^{\frac{1}{2}}-1\right)^{\frac{1}{2}},\left(C^{\frac{1}{2}}-1\right)^{\frac{1}{2}}\right)$. Thus $\lambda_{\alpha} \lambda_{\beta} \leq C^{\frac{1}{2}}-1 \leq v-1$ and moreover

$$
I I_{j \alpha \beta} \geq(3-v)\left(h_{\alpha, \beta j}^{2}+h_{\beta, \alpha j}^{2}\right) .
$$

Lemma 3.1 II $I_{\alpha \beta \gamma} \geq(3-v)\left(h_{\alpha, \beta \gamma}^{2}+h_{\beta, \gamma \alpha}^{2}+h_{\gamma, \alpha \beta}^{2}\right)$.

Proof It is easily seen that

$$
\begin{aligned}
& I I I_{\alpha \beta \gamma}-(3-v)\left(h_{\alpha, \beta \gamma}^{2}+h_{\beta, \gamma \alpha}^{2}+h_{\gamma, \alpha \beta}^{2}\right) \\
& =\left(\lambda_{\alpha} h_{\alpha, \beta \gamma}+\lambda_{\beta} h_{\beta, \gamma \alpha}+\lambda_{\gamma} h_{\gamma, \alpha \beta}\right)^{2}+\left(v-1-\lambda_{\alpha}^{2}\right) h_{\alpha, \beta \gamma}^{2} \\
& \quad+\left(v-1-\lambda_{\beta}^{2}\right) h_{\beta, \gamma \alpha}^{2}+\left(v-1-\lambda_{\gamma}^{2}\right) h_{\gamma, \alpha \beta}^{2} .
\end{aligned}
$$

If $\lambda_{\alpha}^{2}, \lambda_{\beta}^{2}, \lambda_{\gamma}^{2} \leq v-1$, then $I I I_{\alpha \beta \gamma}-(3-v)\left(h_{\alpha, \beta \gamma}^{2}+h_{\beta, \gamma \alpha}^{2}+h_{\gamma, \alpha \beta}^{2}\right)$ is obviously nonnegative definite. Otherwise, we can assume $\lambda_{\gamma}^{2}>v-1$ without loss of generality, then $\left(1+\lambda_{\alpha}^{2}\right)\left(1+\lambda_{\beta}^{2}\right)\left(1+\lambda_{\gamma}^{2}\right) \leq v^{2}$ implies $\lambda_{\alpha}^{2}<v-1, \lambda_{\beta}^{2}<v-1$.

Denote $s=\lambda_{\alpha} h_{\alpha, \beta \gamma}+\lambda_{\beta} h_{\beta, \gamma \alpha}$, then by the Cauchy-Schwarz inequality,

$$
\begin{aligned}
s^{2} & =\left(\lambda_{\alpha} h_{\alpha, \beta \gamma}+\lambda_{\beta} h_{\beta, \gamma \alpha}\right)^{2} \\
& =\left(\frac{\lambda_{\alpha}}{\sqrt{v-1-\lambda_{\alpha}^{2}}} \sqrt{v-1-\lambda_{\alpha}^{2}} h_{\alpha, \beta \gamma}+\frac{\lambda_{\beta}}{\sqrt{v-1-\lambda_{\beta}^{2}}} \sqrt{v-1-\lambda_{\beta}^{2}} h_{\beta, \gamma \alpha}\right)^{2} \\
& \leq\left(\frac{\lambda_{\alpha}^{2}}{v-1-\lambda_{\alpha}^{2}}+\frac{\lambda_{\beta}^{2}}{v-1-\lambda_{\beta}^{2}}\right)\left(\left(v-1-\lambda_{\alpha}^{2}\right) h_{\alpha, \beta \gamma}^{2}+\left(v-1-\lambda_{\beta}^{2}\right) h_{\beta, \gamma \alpha}^{2}\right)
\end{aligned}
$$

i.e.

$$
\left(v-1-\lambda_{\alpha}^{2}\right) h_{\alpha, \beta \gamma}^{2}+\left(v-1-\lambda_{\beta}^{2}\right) h_{\beta, \gamma \alpha}^{2} \geq\left(\frac{\lambda_{\alpha}^{2}}{v-1-\lambda_{\alpha}^{2}}+\frac{\lambda_{\beta}^{2}}{v-1-\lambda_{\beta}^{2}}\right)^{-1} s^{2} .
$$


Hence

$$
\begin{aligned}
& I I I_{\alpha \beta \gamma}-(3-v)\left(h_{\alpha, \beta \gamma}^{2}+h_{\beta, \gamma \alpha}^{2}+h_{\gamma, \alpha \beta}^{2}\right) \\
& \geq\left(s+\lambda_{\gamma} h_{\gamma, \alpha \beta}\right)^{2}+\left(\frac{\lambda_{\alpha}^{2}}{v-1-\lambda_{\alpha}^{2}}+\frac{\lambda_{\beta}^{2}}{v-1-\lambda_{\beta}^{2}}\right)^{-1} s^{2}+\left(v-1-\lambda_{\gamma}^{2}\right) h_{\gamma, \alpha \beta}^{2} \\
& =\left[1+\left(\frac{\lambda_{\alpha}^{2}}{v-1-\lambda_{\alpha}^{2}}+\frac{\lambda_{\beta}^{2}}{v-1-\lambda_{\beta}^{2}}\right)^{-1}\right] s^{2}+(v-1) h_{\gamma, \alpha \beta}^{2}+2 \lambda_{\gamma} s h_{\gamma, \alpha \beta} .
\end{aligned}
$$

It is well known that $a x^{2}+2 b x y+c y^{2}$ is nonnegative definite if and only if $a, c \geq 0$ and $a c-b^{2} \geq 0$. Hence the right hand side of (3.18) is nonnegative definite if and only if

$$
(v-1)\left[1+\left(\frac{\lambda_{\alpha}^{2}}{v-1-\lambda_{\alpha}^{2}}+\frac{\lambda_{\beta}^{2}}{v-1-\lambda_{\beta}^{2}}\right)^{-1}\right]-\lambda_{\gamma}^{2} \geq 0
$$

i.e.

$$
\frac{1}{v-1-\lambda_{\alpha}^{2}}+\frac{1}{v-1-\lambda_{\beta}^{2}}+\frac{1}{v-1-\lambda_{\gamma}^{2}} \leq \frac{2}{v-1} .
$$

Denote $x=1+\lambda_{\alpha}^{2}, y=1+\lambda_{\beta}^{2}, z=1+\lambda_{\gamma}^{2}$. Let $C$ be a constant $\leq v^{2}$, denote

$$
\Omega=\left\{(x, y, z) \in \mathbb{R}^{3}: 1 \leq x, y<v, z>v, x y z=C\right\}
$$

and $f: \Omega \rightarrow \mathbb{R}$

$$
(x, y, z) \mapsto \frac{1}{v-x}+\frac{1}{v-y}+\frac{1}{v-z} .
$$

We claim $f \leq \frac{2}{v-1}$ on $\Omega$. Then (3.20) follows and hence

$$
I I I_{\alpha \beta \gamma}-(3-v)\left(h_{\alpha, \beta \gamma}^{2}+h_{\beta, \gamma \alpha}^{2}+h_{\gamma, \alpha \beta}^{2}\right)
$$

is nonnegative definite.

We now verify the claim. For arbitrary $\varepsilon>0$, denote

$$
f_{\varepsilon}=\frac{1}{v+\varepsilon-x}+\frac{1}{v+\varepsilon-y}+\frac{1}{v+\varepsilon-z},
$$

then $f_{\varepsilon}$ is obviously a smooth function on

$$
\Omega_{\varepsilon}=\left\{(x, y, z) \in \mathbb{R}^{3}: 1 \leq x, y \leq v, z \geq v+2 \varepsilon, x y z=C\right\} .
$$

The compactness of $\Omega_{\varepsilon}$ implies the existence of $\left(x_{0}, y_{0}, z_{0}\right) \in \Omega_{\varepsilon}$ satisfying

$$
f_{\varepsilon}\left(x_{0}, y_{0}, z_{0}\right)=\sup _{\Omega_{\varepsilon}} f_{\varepsilon} .
$$

Fix $x_{0}$, then (3.21) implies that for arbitrary $(y, z) \in \mathbb{R}^{2}$ satisfying $1 \leq y \leq v, z \geq v+2 \varepsilon$ and $y z=\frac{C}{x_{0}}$, we have

$$
f_{\varepsilon, x_{0}}(y, z)=\frac{1}{v+\varepsilon-y}+\frac{1}{v+\varepsilon-z} \leq \frac{1}{v+\varepsilon-y_{0}}+\frac{1}{v+\varepsilon-z_{0}} .
$$


Differentiating both sides of $y z=\frac{C}{x_{0}}$ yields $\frac{d y}{y}+\frac{d z}{z}=0$. Hence

$$
\begin{aligned}
& d\left(\frac{1}{v+\varepsilon-y}+\frac{1}{v+\varepsilon-z}\right)=\frac{d y}{(v+\varepsilon-y)^{2}}+\frac{d z}{(v+\varepsilon-z)^{2}} \\
& =\left[\frac{y}{(v+\varepsilon-y)^{2}}-\frac{z}{(v+\varepsilon-z)^{2}}\right] \frac{d y}{y}=\frac{\left((v+\varepsilon)^{2}-y z\right)(y-z)}{(v+\varepsilon-y)^{2}(v+\varepsilon-z)^{2}} \frac{d y}{y} .
\end{aligned}
$$

It implies that $f_{\varepsilon, x_{0}}\left(y, \frac{C}{y x_{0}}\right)$ is decreasing in $y$ and $y_{0}=1$. Similarly, one can derive $x_{0}=1$. Therefore

$$
\sup _{\Omega_{\varepsilon}} f_{\varepsilon}=f_{\varepsilon}(1,1, C)=\frac{2}{v+\varepsilon-1}+\frac{1}{v+\varepsilon-C}<\frac{2}{v+\varepsilon-1} .
$$

Note that $f_{\varepsilon} \rightarrow f$ and $\Omega \subset \lim _{\varepsilon \rightarrow 0^{+}} \Omega_{\varepsilon}$. Hence by letting $\varepsilon \rightarrow 0$ one can obtain $f \leq \frac{2}{v-1}$.

Lemma 3.2 There exists a positive constant $\varepsilon_{0}$, such that if $v \leq 3$, then

$$
I V_{\alpha} \geq \varepsilon_{0}\left(h_{\alpha, \alpha \alpha}^{2}+\sum_{\beta \neq \alpha}\left(h_{\alpha, \beta \beta}^{2}+2 h_{\beta, \beta \alpha}^{2}\right)\right) .
$$

Proof For arbitrary $\varepsilon_{0} \in[0,1)$, denote $C=1-\varepsilon_{0}$, then

$$
\begin{aligned}
& I V_{\alpha}-\varepsilon_{0}\left(h_{\alpha, \alpha \alpha}^{2}+\sum_{\beta \neq \alpha}\left(h_{\alpha, \beta \beta}^{2}+2 h_{\beta, \beta \alpha}^{2}\right)\right) \\
& =\left(\sum_{\beta} \lambda_{\beta} h_{\beta, \beta \alpha}\right)^{2}+\left(C+\lambda_{\alpha}^{2}\right) h_{\alpha, \alpha \alpha}^{2} \\
& +\sum_{\beta \neq \alpha}\left[C h_{\alpha, \beta \beta}^{2}+\left(2 C+\lambda_{\beta}^{2}\right) h_{\beta, \beta \alpha}^{2}+2 \lambda_{\alpha} \lambda_{\beta} h_{\alpha, \beta \beta} h_{\beta, \beta \alpha}\right] .
\end{aligned}
$$

Obviously

$$
\begin{aligned}
& C h_{\alpha, \beta \beta}^{2}+C^{-1} \lambda_{\alpha}^{2} \lambda_{\beta}^{2} h_{\beta, \beta \alpha}^{2}+2 \lambda_{\alpha} \lambda_{\beta} h_{\alpha, \beta \beta} h_{\beta, \beta \alpha} \\
& \geq\left(C^{\frac{1}{2}} h_{\alpha, \beta \beta}+C^{-\frac{1}{2}} \lambda_{\alpha} \lambda_{\beta} h_{\beta, \beta \alpha}\right)^{2} \geq 0,
\end{aligned}
$$

hence, the third term of the right hand side of (3.23) satisfies

$$
C h_{\alpha, \beta \beta}^{2}+\left(2 C+\lambda_{\beta}^{2}\right) h_{\beta, \beta \alpha}^{2}+2 \lambda_{\alpha} \lambda_{\beta} h_{\alpha, \beta \beta} h_{\beta, \beta \alpha} \geq\left(2 C+\lambda_{\beta}^{2}-C^{-1} \lambda_{\alpha}^{2} \lambda_{\beta}^{2}\right) h_{\beta, \beta \alpha}^{2}
$$

If there exist 2 distinct indices $\beta, \gamma \neq \alpha$ satisfying

$$
2 C+\lambda_{\beta}^{2}-C^{-1} \lambda_{\alpha}^{2} \lambda_{\beta}^{2} \leq 0
$$

and

$$
2 C+\lambda_{\gamma}^{2}-C^{-1} \lambda_{\alpha}^{2} \lambda_{\gamma}^{2} \leq 0
$$


then $\lambda_{\alpha}^{2}>C$ and

$$
\lambda_{\beta}^{2} \geq \frac{2 C^{2}}{\lambda_{\alpha}^{2}-C}, \quad \lambda_{\gamma}^{2} \geq \frac{2 C^{2}}{\lambda_{\alpha}^{2}-C}
$$

It implies

$$
\left(1+\lambda_{\alpha}^{2}\right)\left(1+\lambda_{\beta}^{2}\right)\left(1+\lambda_{\gamma}^{2}\right) \geq \frac{\left(\lambda_{\alpha}^{2}+1\right)\left(\lambda_{\alpha}^{2}+2 C^{2}-C\right)^{2}}{\left(\lambda_{\alpha}^{2}-C\right)^{2}} .
$$

Define $f: x \in(1,+\infty) \mapsto \frac{(x+1)\left(x+2 C^{2}-C\right)^{2}}{(x-C)^{2}}$, then a direct calculation shows

$$
(\log f)^{\prime}=\frac{1}{x+1}+\frac{2}{x+2 C^{2}-C}-\frac{2}{x-C}=\frac{(x-C(2 C+3))(x+C)}{(x+1)\left(x+2 C^{2}-C\right)(x-C)} .
$$

It follows that $f(x) \geq f(C(2 C+3))=\frac{(2 C+1)^{3}}{C+1}$, i.e.

$$
v^{2} \geq\left(1+\lambda_{\alpha}^{2}\right)\left(1+\lambda_{\beta}^{2}\right)\left(1+\lambda_{\gamma}^{2}\right) \geq \frac{(2 C+1)^{3}}{C+1} .
$$

If $C=1$, then $\frac{(2 C+1)^{3}}{C+1}=\frac{27}{2}>9$; hence there is $\varepsilon_{1}>0$, once $\varepsilon_{0} \leq \varepsilon_{1}$, then $C=1-\varepsilon_{0}$ satisfies $\frac{(2 C+1)^{3}}{C+1}>9$, which causes a contradiction to $v^{2} \leq 9$.

Hence, one can find an index $\gamma \neq \alpha$, such that

$$
2 C+\lambda_{\beta}^{2}-C^{-1} \lambda_{\alpha}^{2} \lambda_{\beta}^{2}>0 \text { for arbitrary } \beta \neq \alpha, \gamma .
$$

Denote $s=\sum_{\beta \neq \gamma} \lambda_{\beta} h_{\beta, \beta \alpha}$, then by using the Cauchy-Schwarz inequality,

$$
\begin{aligned}
& \left(C+\lambda_{\alpha}^{2}\right) h_{\alpha, \alpha \alpha}^{2}+\sum_{\beta \neq \alpha, \gamma}\left(2 C+\lambda_{\beta}^{2}-C^{-1} \lambda_{\alpha}^{2} \lambda_{\beta}^{2}\right) h_{\beta, \beta \alpha}^{2} \\
& \geq\left(\frac{\lambda_{\alpha}^{2}}{C+\lambda_{\alpha}^{2}}+\sum_{\beta \neq \alpha, \gamma} \frac{\lambda_{\beta}^{2}}{2 C+\lambda_{\beta}^{2}-C^{-1} \lambda_{\alpha}^{2} \lambda_{\beta}^{2}}\right)^{-1} s^{2} .
\end{aligned}
$$

Substituting (3.27) and (3.24) into (3.23) yields

$$
\begin{aligned}
& I V_{\alpha}-\varepsilon_{0}\left(h_{\alpha, \alpha \alpha}^{2}+\sum_{\beta \neq \alpha}\left(h_{\alpha, \beta \beta}^{2}+2 h_{\beta, \beta \alpha}^{2}\right)\right) \\
& \geq\left(s+\lambda_{\gamma} h_{\gamma, \gamma \alpha}\right)^{2}+\left(\frac{\lambda_{\alpha}^{2}}{C+\lambda_{\alpha}^{2}}+\sum_{\beta \neq \alpha, \gamma} \frac{\lambda_{\beta}^{2}}{2 C+\lambda_{\beta}^{2}-C^{-1} \lambda_{\alpha}^{2} \lambda_{\beta}^{2}}\right)^{-1} s^{2} \\
& \quad+\left(2 C+\lambda_{\gamma}^{2}-C^{-1} \lambda_{\alpha}^{2} \lambda_{\gamma}^{2}\right) h_{\gamma, \gamma \alpha}^{2} \\
& \geq\left[1+\left(\frac{\lambda_{\alpha}^{2}}{C+\lambda_{\alpha}^{2}}+\sum_{\beta \neq \alpha, \gamma} \frac{\lambda_{\beta}^{2}}{2 C+\lambda_{\beta}^{2}-C^{-1} \lambda_{\alpha}^{2} \lambda_{\beta}^{2}}\right)^{-1}\right] s^{2} \\
& \quad+\left(2 C+2 \lambda_{\gamma}^{2}-C^{-1} \lambda_{\alpha}^{2} \lambda_{\gamma}^{2}\right) h_{\gamma, \gamma \alpha}^{2}+2 \lambda_{\gamma} s h_{\gamma, \gamma \alpha} .
\end{aligned}
$$

Note that when $m=2, s=\lambda_{\alpha} h_{\alpha, \alpha \alpha}$ and $\sum_{\beta \neq \alpha, \gamma} \frac{\lambda_{\beta}^{2}}{2 C+\lambda_{\beta}^{2}-C^{-1} \lambda_{\alpha}^{2} \lambda_{\beta}^{2}}=0$. 
The right hand side of (3.28) is nonnegative definite if and only if

$$
2 C+2 \lambda_{\gamma}^{2}-C^{-1} \lambda_{\alpha}^{2} \lambda_{\gamma}^{2} \geq 0
$$

and

$$
\left[1+\left(\frac{\lambda_{\alpha}^{2}}{C+\lambda_{\alpha}^{2}}+\sum_{\beta \neq \alpha, \gamma} \frac{\lambda_{\beta}^{2}}{2 C+\lambda_{\beta}^{2}-C^{-1} \lambda_{\alpha}^{2} \lambda_{\beta}^{2}}\right)^{-1}\right]\left(2 C+2 \lambda_{\gamma}^{2}-C^{-1} \lambda_{\alpha}^{2} \lambda_{\gamma}^{2}\right)-\lambda_{\gamma}^{2} \geq 0 .
$$

Assume $2 C+2 \lambda_{\gamma}^{2}-C^{-1} \lambda_{\alpha}^{2} \lambda_{\gamma}^{2}<0$, then $\lambda_{\alpha}^{2}>2 C$ and $\lambda_{\gamma}^{2}>\frac{2 C^{2}}{\lambda_{\alpha}^{2}-2 C}$, which implies $\left(1+\lambda_{\alpha}^{2}\right)\left(1+\lambda_{\gamma}^{2}\right) \geq \frac{\left(\lambda_{\alpha}^{2}+1\right)\left(\lambda_{\alpha}^{2}+2 C(C-1)\right)}{\lambda_{\alpha}^{2}-2 C}$. Define $f: x \in(2 C,+\infty) \mapsto \frac{(x+1)(x+2 C(C-1))}{x-2 C}$, then

$$
(\log f)^{\prime}=\frac{1}{x+1}+\frac{1}{x+2 C(C-1)}-\frac{1}{x-2 C}=\frac{x^{2}-4 C x-2 C^{2}(2 C-1)}{(x+1)(x+2 C(C-1))(x-2 C)} .
$$

and hence

$$
\min f=f(C(2+\sqrt{4 C+2}))=2 C^{2}+2 C+1+2 C \sqrt{4 C+2} .
$$

In particular, when $C=1, \min f=5+2 \sqrt{6}>9$. There exists $\varepsilon_{2}>0$, such that once $\varepsilon_{0} \leq \varepsilon_{2}$, one can derive min $f>9$ and moreover $v^{2} \geq\left(1+\lambda_{\alpha}^{2}\right)\left(1+\lambda_{\gamma}^{2}\right)>9$, which contradicts $v \leq 3$. Therefore (3.29) holds.

If $2 C+\lambda_{\gamma}^{2}-C^{-1} \lambda_{\alpha}^{2} \lambda_{\gamma}^{2} \geq 0$, (3.30) trivially holds.

At last, we consider the situation when there exists $\gamma, \gamma \neq \alpha$, such that

$$
2 C+\lambda_{\gamma}^{2}-C^{-1} \lambda_{\alpha}^{2} \lambda_{\gamma}^{2}<0
$$

In this case, (3.30) is equivalent to

$$
\frac{\lambda_{\alpha}^{2}}{C+\lambda_{\alpha}^{2}}+\sum_{\beta \neq \alpha} \frac{\lambda_{\beta}^{2}}{2 C+\lambda_{\beta}^{2}-C^{-1} \lambda_{\alpha}^{2} \lambda_{\beta}^{2}} \leq-1
$$

Noting that

$$
\frac{\lambda_{\beta}^{2}}{2 C+\lambda_{\beta}^{2}-C^{-1} \lambda_{\alpha}^{2} \lambda_{\beta}^{2}}=\frac{C}{C-\lambda_{\alpha}^{2}}-\frac{2 C^{3}}{\left(C-\lambda_{\alpha}^{2}\right)^{2}} \frac{1}{1+\lambda_{\beta}^{2}+\frac{\lambda_{\alpha}^{2}+C(2 C-1)}{C-\lambda_{\alpha}^{2}}}
$$

and let $x_{\beta}=1+\lambda_{\beta}^{2}$, then (3.31) is equivalent to

$$
\frac{x_{\alpha}-1}{x_{\alpha}+C-1}+\sum_{\beta \neq \alpha}\left[\frac{C}{C+1-x_{\alpha}}-\frac{2 C^{3}}{\left(C+1-x_{\alpha}\right)^{2}} \frac{1}{x_{\beta}-\frac{x_{\alpha}+2 C^{2}-C-1}{x_{\alpha}-C-1}}\right] \leq-1 .
$$

Denote

$$
\begin{aligned}
& \psi\left(x_{\alpha}\right)=\frac{x_{\alpha}-1}{x_{\alpha}+C-1}, \quad \varphi\left(x_{\alpha}\right)=\frac{x_{\alpha}+2 C^{2}-C-1}{x_{\alpha}-C-1}, \\
& \zeta\left(x_{\alpha}\right)=\frac{C}{C+1-x_{\alpha}}, \quad \xi\left(x_{\alpha}\right)=\frac{2 C^{3}}{\left(C+1-x_{\alpha}\right)^{2}} .
\end{aligned}
$$


Let

$$
\begin{aligned}
& \Omega=\left\{\begin{array}{l}
\left(x_{1}, \ldots, x_{m}\right) \in \mathbb{R}^{m}: x_{\alpha}>C+1,1 \leq x_{\beta}<\varphi\left(x_{\alpha}\right) \text { for all } \beta \neq \alpha, \gamma, \\
x_{\gamma}>\varphi\left(x_{\alpha}\right), \prod_{\beta} x_{\beta}=v^{2}
\end{array}\right\}
\end{aligned}
$$

and define $f: \Omega \rightarrow \mathbb{R}$

$$
\left(x_{1}, \ldots, x_{m}\right) \mapsto \psi\left(x_{\alpha}\right)+\sum_{\beta \neq \alpha}\left[\zeta\left(x_{\alpha}\right)-\frac{\xi\left(x_{\alpha}\right)}{x_{\beta}-\varphi\left(x_{\alpha}\right)}\right] .
$$

We point out that in (3.33), $\alpha$ and $\gamma$ are fixed indices.

Now we claim

$$
\sup _{\Omega} f=\sup _{\Gamma} f
$$

where

$$
\begin{aligned}
& \Gamma=\left\{\left(x_{1}, \ldots, x_{m}\right) \in \mathbb{R}^{m}: x_{\alpha} \geq C+1, x_{\beta}=1 \text { for all } \beta \neq \alpha, \gamma,\right. \\
& \left.x_{\gamma} \geq \varphi\left(x_{\alpha}\right), \prod_{\beta} x_{\beta}=v^{2}\right\} \subset \Omega .
\end{aligned}
$$

When $m=2$, obviously $\Gamma=\Omega$ and (3.34) is trivial. We put

$$
\varphi_{\varepsilon}\left(x_{\alpha}\right)=\varphi\left(x_{\alpha}+\varepsilon\right), \zeta_{\varepsilon}\left(x_{\alpha}\right)=\zeta\left(x_{\alpha}+\varepsilon\right), \xi_{\varepsilon}\left(x_{\alpha}\right)=\xi\left(x_{\alpha}+\varepsilon\right)
$$

for arbitrary $\varepsilon>0$. If $m \geq 3$, as in the proof of Lemma 3.1, we define

$$
f_{\varepsilon}=\psi\left(x_{\alpha}\right)+\sum_{\beta \neq \alpha}\left[\zeta_{\varepsilon}\left(x_{\alpha}\right)-\frac{\xi_{\varepsilon}\left(x_{\alpha}\right)}{x_{\beta}-\varphi_{\varepsilon}\left(x_{\alpha}\right)}\right],
$$

then $f_{\varepsilon}$ is well-defined on

$$
\begin{aligned}
\Omega_{\varepsilon}= & \left\{\left(x_{1}, \ldots, x_{m}\right) \in \mathbb{R}^{m}: x_{\alpha} \geq C+1,1 \leq x_{\beta} \leq \varphi_{2 \varepsilon}\left(x_{\alpha}\right) \text { for all } \beta \neq \alpha, \gamma,\right. \\
& \left.x_{\gamma} \geq \varphi_{\frac{\varepsilon}{2}}\left(x_{\alpha}\right), \prod_{\beta} x_{\beta}=v^{2}\right\} .
\end{aligned}
$$

The compactness of $\Omega_{\varepsilon}$ enables us to find $\left(y_{1}, \ldots, y_{m}\right) \in \Omega_{\varepsilon}$, such that

$$
f_{\varepsilon}\left(y_{1}, \ldots, y_{m}\right)=\sup _{\Omega_{\varepsilon}} f_{\varepsilon} .
$$

Denote $b=\varphi_{\varepsilon}\left(y_{\alpha}\right)$, then (3.36) implies for arbitrary $\beta \neq \alpha, \gamma$ that

$$
\frac{1}{x_{\beta}-b}+\frac{1}{x_{\gamma}-b} \geq \frac{1}{y_{\beta}-b}+\frac{1}{y_{\gamma}-b}
$$


holds whenever $x_{\beta} x_{\gamma}=y_{\beta} y_{\gamma}, 1 \leq x_{\beta} \leq \varphi_{2 \varepsilon}\left(y_{\alpha}\right)$ and $x_{\gamma} \geq \varphi_{\frac{\varepsilon}{2}}\left(y_{\alpha}\right)$. Differentiating both sides yields $\frac{d x_{\beta}}{x_{\beta}}+\frac{d x_{\gamma}}{x_{\gamma}}=0$, thus

$$
d\left(\frac{1}{x_{\beta}-b}+\frac{1}{x_{\gamma}-b}\right)=-\frac{d x_{\beta}}{\left(x_{\beta}-b\right)^{2}}-\frac{d x_{\gamma}}{\left(x_{\gamma}-b\right)^{2}}=\frac{\left(b^{2}-x_{\beta} x_{\gamma}\right)\left(x_{\gamma}-x_{\beta}\right)}{\left(x_{\beta}-b\right)^{2}\left(x_{\gamma}-b\right)^{2}} \frac{d x_{\beta}}{x_{\beta}} .
$$

Similarly to (3.25), one can prove $y_{\alpha} b^{2}=\frac{y_{\alpha}\left(y_{\alpha}+\varepsilon+2 C^{2}-C-1\right)^{2}}{\left(y_{\alpha}+\varepsilon-C-1\right)^{2}}>9$ when $\varepsilon_{0} \leq \varepsilon_{1}$ (note that $\left.C=1-\varepsilon_{0}\right)$ and $\varepsilon_{1}$ is sufficiently small. In conjunction with $y_{\alpha} x_{\beta} x_{\gamma}=y_{\alpha} y_{\beta} y_{\gamma} \leq v^{2}<9$, we have $b^{2}-x_{\beta} x_{\gamma}>0$. Hence (3.37) implies $y_{\beta}=1$ for all $\beta \neq \alpha, \gamma$. In other words, if we put

$$
\begin{aligned}
\Gamma_{\varepsilon}=\{ & \left(x_{1}, \ldots, x_{m}\right) \in \mathbb{R}^{m}: x_{\alpha} \geq C+1, x_{\beta}=1 \text { for all } \beta \neq \alpha, \gamma, \\
& \left.x_{\gamma} \geq \varphi_{\frac{\varepsilon}{2}}\left(x_{\alpha}\right), \prod_{\beta} x_{\beta}=v^{2}\right\},
\end{aligned}
$$

then $\max _{\Omega_{\varepsilon}} f_{\varepsilon}=\max _{\Gamma_{\varepsilon}} f_{\varepsilon}$. Therefore, (3.34) follows from $\Omega \subset \bigcup_{\varepsilon>0} \Omega_{\varepsilon}, \Gamma \subset \bigcup_{\varepsilon>0} \Gamma_{\varepsilon}$ and $\lim _{\varepsilon \rightarrow 0} f_{\varepsilon}=f$.

To prove (3.30), i.e. $f \leq-1$, it is sufficient to show on $\Gamma$,

$$
\psi\left(x_{\alpha}\right)+\zeta\left(x_{\alpha}\right)-\frac{\xi\left(x_{\alpha}\right)}{\frac{v^{2}}{x_{\alpha}}-\varphi\left(x_{\alpha}\right)} \leq-1
$$

whenever $x_{\alpha}>C+1$ and $\frac{v^{2}}{x_{\alpha}}>\varphi\left(x_{\alpha}\right)$. After a straightforward calculation, the above inequality is equivalent to

$$
\begin{gathered}
x_{\alpha}^{3}+\left(2 C^{2}-C-2\right) x_{\alpha}^{2}+\left(C^{3}-3 C^{2}+C+1\right) x_{\alpha} \\
-v^{2}\left(x_{\alpha}^{2}-(C+2) x_{\alpha}-\left(C^{2}-C-1\right)\right) \geq 0 .
\end{gathered}
$$

It is easily seen that if

$$
\inf _{t^{2}-(C+2) t-\left(C^{2}-C-1\right)>0} \frac{t^{3}+\left(2 C^{2}-C-2\right) t^{2}+\left(C^{3}-3 C^{2}+C+1\right) t}{t^{2}-(C+2) t-\left(C^{2}-C-1\right)}>9 .
$$

then (3.39) naturally holds and furthermore one can deduce that $I V_{\alpha}-\varepsilon_{0}\left(h_{\alpha, \alpha \alpha}^{2}+\sum_{\beta \neq \alpha}\right.$ $\left.\left(h_{\alpha, \beta \beta}^{2}+2 h_{\beta, \beta \alpha}^{2}\right)\right)$ is nonnegative definite.

When $C=1$, (3.40) becomes

$$
\inf _{t>\frac{3+\sqrt{5}}{2}} \frac{t^{2}(t-1)}{t^{2}-3 t+1}>9 .
$$

If this is true, one can find a positive constant $\varepsilon_{3}$ to ensure (3.40) holds true whenever $\varepsilon_{0} \leq \varepsilon_{3}$. Finally, by taking $\varepsilon_{0}=\min \left\{\varepsilon_{1}, \varepsilon_{2}, \varepsilon_{3}\right\}$ we obtain the final conclusion.

(3.41) is equivalent to the property that $h(t)=t^{2}(t-1)-9\left(t^{2}-3 t+1\right)=t^{3}-10 t^{2}+27 t-9$ has no zeros on $\left(\frac{3+\sqrt{5}}{2},+\infty\right) \cdot h^{\prime}(t)=3 t^{2}-20 t+27$ implies $h^{\prime}(t)<0$ on $\left(\frac{3+\sqrt{5}}{2}, \frac{10+\sqrt{19}}{3}\right)$ and $h^{\prime}(t)>0$ on $\left(\frac{10+\sqrt{19}}{3},+\infty\right)$, hence 


$$
\inf _{t>\frac{3+\sqrt{5}}{2}} h=h\left(\frac{10+\sqrt{19}}{3}\right)=\frac{187-38 \sqrt{19}}{27}>0
$$

and (3.41) follows.

In conjunction with (3.13), (3.16), Lemma 3.1 and 3.2, we can arrive at

Theorem 3.1 Let $M^{n}$ be a submanifold in $\mathbb{R}^{n+m}$ with parallel mean curvature, then for arbitrary $p \in M$ and $P_{0} \in \mathbf{G}_{n, m}$, once $v\left(\gamma(p), P_{0}\right) \leq 3$, then $\Delta\left(v\left(\cdot, P_{0}\right) \circ \gamma\right) \geq 0$ at $p$. Moreover, if $v\left(\gamma(p), P_{0}\right) \leq \beta_{0}<3$, then there exists a positive constant $K_{0}$, depending only on $\beta_{0}$, such that

$$
\Delta\left(v\left(\cdot, P_{0}\right) \circ \gamma\right) \geq K_{0}|B|^{2}
$$

at $p$.

We also express this result by saying that the function $v$ satisfying (3.42) is strongly subharmonic under the condition $v\left(\gamma(p), P_{0}\right) \leq \beta_{0}<3$.

Remark 3.1 If $\log v$ is a strongly subharmonic function, then $v$ is certainly strongly subharmonic, but the converse is not necessarily true. Therefore, the above result does not seem to follow from Theorem 1.2 in [18].

\section{Curvature estimates}

Let $z^{\alpha}=f^{\alpha}\left(x^{1}, \ldots, x^{n}\right), \alpha=1, \ldots, m$ be smooth functions defined on $D_{R_{0}} \subset \mathbb{R}^{n}$. Their graph $M=(x, f(x))$ is a submanifold with parallel mean curvature in $\mathbb{R}^{n+m}$. Suppose there is $\beta_{0} \in[1,3)$, such that

$$
\Delta_{f}=\left[\operatorname{det}\left(\delta_{i j}+\sum_{\alpha} \frac{\partial f^{\alpha}}{\partial x^{i}} \frac{\partial f^{\alpha}}{\partial x^{j}}\right)\right]^{\frac{1}{2}} \leq \beta_{0} .
$$

Denote by $\epsilon_{1}, \ldots, \epsilon_{n+m}$ the canonical basis of $\mathbb{R}^{n+m}$ and put $P_{0}=\epsilon_{1} \wedge \cdots \wedge \epsilon_{n}$. Then by (4.1)

$$
v\left(\cdot, P_{0}\right) \circ \gamma \leq \beta_{0}
$$

holds everywhere on $M$. Putting $v=v\left(\cdot, P_{0}\right) \circ \gamma$, Theorem 3.1 tells us

$$
\Delta v \geq K_{0}\left(\beta_{0}\right)|B|^{2} .
$$

Let $\eta$ be a nonnegative smooth function on $M$ with compact support. Multiplying both sides of (4.2) by $\eta$ and integrating on $M$ gives

$$
K_{0} \int_{M}|B|^{2} \eta * 1 \leq-\int_{M} \nabla \eta \cdot \nabla v * 1 .
$$

$F: D_{R_{0}} \mapsto M$ defined by

$$
x=\left(x^{1}, \ldots, x^{n}\right) \mapsto(x, f(x))
$$

is obviously a diffeomorphism. $F_{*} \frac{\partial}{\partial x^{i}}=\epsilon_{i}+\frac{\partial f^{\alpha}}{\partial x^{i}} \epsilon_{n+\alpha}$ implies

$$
\left\langle F_{*} \frac{\partial}{\partial x^{i}}, F_{*} \frac{\partial}{\partial x^{j}}\right\rangle=\delta_{i j}+\sum_{\alpha} \frac{\partial f^{\alpha}}{\partial x^{i}} \frac{\partial f^{\alpha}}{\partial x^{j}} .
$$


Hence

$$
F^{*} g=\left(\delta_{i j}+\sum_{\alpha} \frac{\partial f^{\alpha}}{\partial x^{i}} \frac{\partial f^{\alpha}}{\partial x^{j}}\right) d x^{i} d x^{j}
$$

where $g$ is the metric tensor on $M$. In other words, $M$ is isometric to the Euclidean ball $D_{R_{0}}$ equipped with the metric $g_{i j} d x^{i} d x^{j}\left(g_{i j}=\delta_{i j}+\sum_{\alpha} \frac{\partial f^{\alpha}}{\partial x^{i}} \frac{\partial f^{\alpha}}{\partial x^{j}}\right)$. It is easily seen that for arbitrary $\xi \in \mathbb{R}^{n}$,

$$
\xi^{i} g_{i j} \xi^{j}=|\xi|^{2}+\sum_{\alpha}\left(\sum_{i} \frac{\partial f^{\alpha}}{\partial x^{i}} \xi^{i}\right)^{2} \geq|\xi|^{2} .
$$

On the other hand, $\Delta_{f} \leq \beta_{0}$ implies $\prod_{i=1}^{n} \mu_{i} \leq \beta_{0}^{2}$, with $\mu_{1}, \ldots, \mu_{n}$ the eigenvalues of $\left(g_{i j}\right)$, thus

$$
\xi^{i} g_{i j} \xi^{j} \leq \beta_{0}^{2}|\xi|^{2} \leq 9|\xi|^{2}
$$

In local coordinates, the Laplace-Beltrami operator is

$$
\Delta=\frac{1}{\sqrt{G}} \frac{\partial}{\partial x^{i}}\left(\sqrt{G} g^{i j} \frac{\partial}{\partial x^{j}}\right) .
$$

Here $\left(g^{i j}\right)$ is the inverse matrix of $\left(g_{i j}\right)$, and $G=\operatorname{det}\left(g_{i j}\right)=\Delta_{f}^{2}$. From (4.1), (4.5) and (4.6) it is easily seen that

$$
\frac{1}{3}|\xi|^{2} \leq \beta_{0}^{-1}|\xi|^{2} \leq \xi^{i}\left(\sqrt{G} g^{i j}\right) \xi^{j} \leq \beta_{0}|\xi|^{2} \leq 3|\xi|^{2} .
$$

Following $[11,13]$ we shall make use of the following abbreviations: For arbitrary $R \in$ $\left(0, R_{0}\right)$, let

$$
B_{R}=\left\{(x, f(x)): x \in D_{R}\right\} \subset M .
$$

And for arbitrary $h \in L^{\infty}\left(B_{R}\right)$ denote

$$
\begin{aligned}
& h_{+, R} \stackrel{\text { def. }}{=} \sup _{B_{R}} h, \quad h_{-, R} \stackrel{\text { def. }}{=}=\inf _{B_{R}} h, \quad \bar{h}_{R} \stackrel{\text { def. }}{=} f_{B_{R}} h=\frac{\int_{B_{R}} h * 1}{\left|\operatorname{Vol}\left(B_{R}\right)\right|} \\
& |\bar{h}|_{p, R} \stackrel{\text { def. }}{=}\left(f_{B_{R}}|h|^{p}\right)^{\frac{1}{p}}(p \in(-\infty,+\infty)) .
\end{aligned}
$$

(4.7) shows that $\Delta$ is a uniform elliptic operator. Moser's Harnack inequality [15] for supersolutions of elliptic PDEs in divergence form gives

Lemma 4.1 For a positive superharmonic function $h$ on $B_{R}$ with $R \in\left(0, R_{0}\right], p \in\left(0, \frac{n}{n-2}\right)$ and $\theta \in\left[\frac{1}{2}, 1\right)$, we have the following estimate

$$
|\bar{h}|_{p, \theta R} \leq \gamma_{1} h_{-, \theta R} .
$$

Here $\gamma_{1}$ is a positive constant only depending on $n, p$ and $\theta$, but not on $h$ and $R$.

(4.2) shows the subharmonicity of $v$, and therefore $v_{+, R}-v+\varepsilon$ is a positive superharmonic function on $B_{R}$ for arbitrary $\varepsilon>0$. With the aid of Lemma 4.1, one can follow [11] to get 
Corollary 4.1 There is a constant $\delta_{0} \in(0,1)$, depending only on $n$, such that

$$
v_{+, \frac{R}{2}} \leq\left(1-\delta_{0}\right) v_{+, R}+\delta_{0} \bar{v}_{\frac{R}{2}} .
$$

Denote by $G^{\rho}$ the mollified Green function for the Laplace-Beltrami operator on $B_{R}$. Then for arbitrary $p=(y, f(y)) \in B_{R}$, once

$$
B_{\rho}(p)=\left\{(x, f(x)) \in M: x \in D_{R}(y)\right\} \subset B_{R}
$$

we have

$$
\int_{B_{R}} \nabla G^{\rho}(\cdot, p) \cdot \nabla \phi * 1=\underset{B_{\rho}(p)}{f_{p} \phi}
$$

for every $\phi \in H_{0}^{1,2}\left(B_{R}\right)$. The a priori estimates for mollified Green functions of [8] tell us

Lemma 4.2 With $o:=(0, f(0))$, we have

$$
\begin{aligned}
0 \leq G^{\frac{R}{2}}(\cdot, o) \leq c_{2}(n) R^{2-n} & \text { on } B_{R} \\
G^{\frac{R}{2}}(\cdot, o) \geq c_{1}(n) R^{2-n} & \text { on } B_{\frac{R}{2}} .
\end{aligned}
$$

For arbitrary $p \in B_{\frac{R}{4}}$,

$$
G^{\rho}(\cdot, p) \leq C(n) R^{2-n} \text { on } B_{R} \backslash \bar{B}_{\frac{R}{2}} .
$$

Moreover if $\rho \leq \frac{R}{8}$,

$$
\int_{B_{R} \backslash \bar{B}_{\frac{R}{2}}}\left|\nabla G^{\rho}(\cdot, p)\right|^{2} * 1 \leq C(n) R^{2-n} .
$$

Based on (4.3), Corollary 4.1 and Lemma 4.2, we can use the method of [11] to derive a telescoping lemma a la Giaquinta and Giusti [6] and Giaquita and Hildebrandt [7].

Theorem 4.1 There exists a positive constant $C_{1}$, only depending on $n$ and $\beta_{0}$, such that for arbitrary $R \leq R_{0}$,

$$
R^{2-n} \int_{B_{\frac{R}{2}}}|B|^{2} * 1 \leq C_{1}\left(v_{+, R}-v_{+, \frac{R}{2}}\right)
$$

Moreover, there exists a positive constant $C_{2}$, only depending on $n$ and $\beta_{0}$, such that for arbitrary $\varepsilon>0$, we can find $R \in\left[\exp \left(-C_{2} \varepsilon^{-1}\right) R_{0}, R_{0}\right]$, such that

$$
R^{2-n} \int_{B_{\frac{R}{2}}}|B|^{2} * 1 \leq \varepsilon
$$

Proof With

$$
\omega^{R}=R^{-2} \operatorname{Vol}\left(B_{\frac{R}{2}}\right) G^{\frac{R}{2}}(\cdot, o) \text { where } o=(0, f(0)),
$$


then

$$
\int_{B_{R}} \nabla \omega^{R} \cdot \nabla \phi * 1=R^{-2} \int_{B_{\frac{R}{2}}} \phi * 1 .
$$

Choosing $\left(\omega^{R}\right)^{2} \in H_{0}^{1,2}\left(B_{R}\right)$ as a test function in (4.3), we obtain

$$
\begin{aligned}
K_{0} \int_{B_{R}}|B|^{2}\left(\omega^{R}\right)^{2} * 1 & \leq-\int_{B_{R}} \nabla\left(\omega^{R}\right)^{2} \cdot \nabla v * 1=-2 \int_{B_{R}} \nabla \omega^{R} \cdot \omega^{R} \nabla\left(v-v_{+, R}\right) * 1 \\
& =-2 \int_{B_{R}} \nabla \omega^{R} \cdot\left(\nabla\left(\omega^{R}\left(v-v_{+, R}\right)\right)-\left(v-v_{+, R}\right) \nabla \omega^{R}\right) * 1 \\
& \leq-2 \int_{B_{R}} \nabla \omega^{R} \cdot \nabla\left(\omega^{R}\left(v-v_{+, R}\right)\right) * 1 \\
& =-2 R^{-2} \int_{B_{\frac{R}{2}}} \omega^{R}\left(v-v_{+, R}\right) * 1 .
\end{aligned}
$$

By (4.10), there exist positive constants $c_{3}, c_{4}$, depending only on $n$, such that

$$
\begin{aligned}
0 \leq \omega^{R} \leq c_{4} & \text { on } B_{R}, \\
\omega^{R} \geq c_{3} & \text { on } B_{\frac{R}{2}} .
\end{aligned}
$$

Hence

$$
\begin{aligned}
\int_{B_{\frac{R}{2}}}|B|^{2} * 1 & \leq-2 K_{0}^{-1} c_{4}^{-1} c_{3}^{2} R^{-2} \int_{B_{\frac{R}{2}}}\left(v-v_{+, R}\right) * 1 \\
& \leq c_{5}\left(n, \beta_{0}\right) R^{n-2}\left(v_{+, R}-\bar{v}_{\frac{R}{2}}\right) .
\end{aligned}
$$

By Corollary 4.1, $v_{+, R}-\bar{v}_{\frac{R}{2}} \leq \delta_{0}^{-1}\left(v_{+, R}-v_{+, \frac{R}{2}}\right)$; substituting it into (4.15) yields (4.13).

For arbitrary $k \in \mathbb{Z}^{+},(4.13)$ gives

$$
\begin{aligned}
\sum_{i=0}^{k}\left(2^{-i} R_{0}\right)^{2-n} \int_{B_{2-i-1} R_{0}}|B|^{2} * 1 & \leq C_{1} \sum_{i=0}^{k}\left(v_{+, 2^{-i} R_{0}}-v_{+, 2^{-i-1} R_{0}}\right) \\
& =C_{1}\left(v_{+, R_{0}}-v_{+, 2^{-k-1} R_{0}}\right) \\
& \leq C_{1}\left(\beta_{0}-1\right) \leq 2 C_{1}
\end{aligned}
$$

For arbitrary $\varepsilon>0$, we take

$$
k=\left[2 C_{1} \varepsilon^{-1}\right],
$$

then we can find $1 \leq j \leq k$, such that

$$
\left(2^{-j} R_{0}\right)^{2-n} \int_{B_{2^{-j-1} R_{0}}}|B|^{2} * 1 \leq \frac{2}{k+1} C_{1} \leq \varepsilon .
$$


Since $2^{-j} \geq 2^{-k} \geq 2^{-2 C_{1} \varepsilon^{-1}}=\exp \left[-2(\log 2) C_{1} \varepsilon^{-1}\right]$, it is sufficient to choose $C_{2}=$ $-2(\log 2) C_{1}$.

\section{Gauss image shrinking property}

Lemma 5.1 For arbitrary $a>1$ and $\beta_{0} \in[1, a)$, there exists a positive constant $\varepsilon_{1}=$ $\varepsilon_{1}\left(a, \beta_{0}\right)$ with the following property. If $P_{1}, Q \in \mathbf{G}_{n, m}$ satisfies $v\left(Q, P_{1}\right) \leq b \leq \beta_{0}$, then we can find $P_{2} \in \mathbf{G}_{n, m}$, such that $v\left(P, P_{2}\right) \leq$ a for every $P \in \mathbf{G}_{n, m}$ satisfying $v\left(P, P_{1}\right) \leq b$, and

$$
1 \leq v\left(Q, P_{2}\right) \leq \begin{cases}1 & \text { if } b<\sqrt{2}\left(1+a^{-1}\right)^{-\frac{1}{2}} \\ b-\varepsilon_{1} & \text { otherwise. }\end{cases}
$$

Proof Obviously $w(P, P)=1$ for every $P \in \mathbf{G}_{n, m}$, which shows $\mathbf{G}_{n, m}$ is a submanifold in a Euclidean sphere via the Plücker embedding. Denote by $r(\cdot, \cdot)$ the restriction of the spherical distance on $\mathbf{G}_{n, m}$, then by spherical geometry, $w=\cos r$ and hence $v=\sec r$.

Denote $\alpha=\arccos \left(a^{-1}\right)$ and $\beta=\arccos \left(b^{-1}\right)$. Now we put $\gamma=\alpha-\beta$ and

$$
c=\sec \gamma=\left(a^{-1} b^{-1}+\left(1-a^{-2}\right)^{\frac{1}{2}}\left(1-b^{-2}\right)^{\frac{1}{2}}\right)^{-1} .
$$

Once $v\left(P_{2}, P_{1}\right) \leq c$, the triangle inequality implies

$$
r\left(P, P_{2}\right) \leq r\left(P, P_{1}\right)+r\left(P_{2}, P_{1}\right) \leq \arccos \left(b^{-1}\right)+\arccos \left(c^{-1}\right)=\alpha
$$

for every $P$ satisfying $v\left(P, P_{1}\right) \leq b$, and thus $v\left(P, P_{2}\right) \leq a$ follows.

If $b<\sqrt{2}\left(1+a^{-1}\right)^{-\frac{1}{2}}$, then a direct calculation shows $\beta<\frac{\alpha}{2}$, hence $\gamma>\beta$ and moreover $v\left(Q, P_{1}\right) \leq b<c$. Thereby $P_{2}=Q$ is the required point.

Otherwise $b \geq \sqrt{2}\left(1+a^{-1}\right)^{-\frac{1}{2}}$ and hence $c \leq b$. Obviously one of the following two cases has to occur:

Case I. $v\left(Q, P_{1}\right)<c$. One can take $P_{2}=Q$ to ensure $v\left(\cdot, P_{2}\right) \leq a$ whenever $v\left(\cdot, P_{1}\right) \leq b$. In this case

$$
b-v\left(Q, P_{2}\right)=b-1 \geq \sqrt{2}\left(1+a^{-1}\right)^{-\frac{1}{2}}-1 .
$$

Case II. $v\left(Q, P_{1}\right) \geq c$. Denote by $\theta_{1}, \ldots, \theta_{m}$ the Jordan angles between $Q$ and $P_{1}$, and put $L^{2}=\sum_{1 \leq \alpha \leq m} \theta_{\alpha}^{2}$, then as shown in [19], if we denote the shortest normal geodesic from $Q$ to $P_{1}$ by $\gamma$, then the Jordan angles between $Q$ and $\gamma(t)$ are $\frac{\theta_{1}}{L} t, \ldots, \frac{\theta_{m}}{L} t$, while the Jordan angles between $\gamma(t)$ and $P_{1}$ are $\frac{\theta_{1}}{L}(L-t), \ldots, \frac{\theta_{m}}{L}(L-t)$. Hence

$$
\begin{aligned}
v(Q, \gamma(t)) & =\prod_{\alpha} \sec \left(\frac{\theta_{\alpha}}{L} t\right), \\
v\left(\gamma(t), P_{1}\right) & =\prod_{\alpha} \sec \left(\frac{\theta_{\alpha}}{L}(L-t)\right) .
\end{aligned}
$$

Since $t \mapsto \prod_{\alpha} \sec \left(\frac{\theta_{\alpha}}{L}(L-t)\right)$ is a strictly decreasing function, there exists a unique $t_{0} \in$ $[0, L)$, such that $\prod_{\alpha} \sec \left(\frac{\theta_{\alpha}}{L}\left(L-t_{0}\right)\right)=c$. Now we choose $P_{2}=\gamma\left(t_{0}\right)$, then $v\left(P_{2}, P_{1}\right)=c$ and

$$
b-v\left(Q, P_{2}\right)=b-\prod_{\alpha} \sec \left(\frac{\theta_{\alpha}}{L} t_{0}\right) .
$$


It remains to show $b-\prod_{\alpha} \sec \left(\frac{\theta_{\alpha}}{L} t_{0}\right)$ is bounded from below by a universal positive constant $\varepsilon_{2}$. Once this holds true, in conjunction with (5.3) and (5.4),

$$
\varepsilon_{1}=\min \left\{\sqrt{2}\left(1+a^{-1}\right)^{-\frac{1}{2}}-1, \varepsilon_{2}\right\}
$$

is the required constant.

$t_{0}$ can be regarded as a smooth function on

$$
\Omega=\left\{\left(b, \theta_{1}, \ldots, \theta_{m}\right) \in \mathbb{R}^{m+1}, \sqrt{2}\left(1+a^{-1}\right)^{-\frac{1}{2}} \leq b \leq \beta_{0}, 0 \leq \theta_{\alpha} \leq \frac{\pi}{2}, c \leq \prod_{\alpha} \sec \left(\theta_{\alpha}\right) \leq b\right\}
$$

which is the unique one satisfying

$$
\prod_{\alpha} \sec \left(\frac{\theta_{\alpha}}{L}\left(L-t_{0}\right)\right)=c .
$$

(By (5.2), $c$ can be viewed as a function of $b$.) The smoothness of $t_{0}$ follows from the implicit function theorem. Therefore $F: \Omega \rightarrow \mathbb{R}$

$$
\left(\theta_{1}, \ldots, \theta_{m}\right) \mapsto b-\prod_{\alpha} \sec \left(\frac{\theta_{\alpha}}{L} t_{0}\right)
$$

is a smooth function on $\Omega$. $t_{0}<L$ implies $F>0$; then the compactness of $\Omega$ gives $\inf _{\Omega} F>0$, and $\varepsilon_{2}=\inf _{\Omega} F$ is the required constant.

Remark $5.1 \varepsilon_{1}$ is only depending on $a$ and $\beta_{0}$, non-decreasingly during the iteration process in Theorem 6.1.

Theorem 5.1 Let $M=\left\{(x, f(x)): x \in D_{R_{0}} \subset \mathbb{R}^{n}\right\}$ be a graph with parallel mean curvature, and $\Delta_{f} \leq \beta_{0}$ with $\beta_{0} \in[1,3)$. Assume there exists $P_{0} \in \mathbf{G}_{n, m}$, such that $v\left(\cdot, P_{0}\right) \circ \gamma \leq b$ on $M$ with $1 \leq b \leq \beta_{0}$. If $b<\frac{\sqrt{6}}{2}$, then for arbitrary $\varepsilon>0$, one can find a constant $\delta \in(0,1)$ depending only on $n, \beta_{0}$ and $\varepsilon$ such that

$$
1 \leq v\left(\cdot, P_{1}\right) \circ \gamma \leq 1+\varepsilon \text { on } B_{\delta R_{0}}
$$

for a point $P_{1} \in \mathbf{G}_{n, m}$. If $b \geq \frac{\sqrt{6}}{2}$, then there are two constants $\delta_{0} \in(0,1)$ and $\varepsilon_{1}>0$, only depending on $n$ and $\beta_{0}$, such that

$$
1 \leq v\left(\cdot, P_{1}\right) \circ \gamma \leq b-\frac{\varepsilon_{1}}{2} \text { on } B_{\delta_{0} R_{0}}
$$

for a point $P_{1} \in \mathbf{G}_{n, m}$.

Proof Let $H$ be a smooth function on $\mathbf{G}_{n, m}$, then $h=H \circ \gamma$ gives a smooth function on $M$. Let $\eta$ be a nonnegative smooth function on $M$ with compact support and $\varphi$ be a $H^{1,2}$-function on $M$, then by Stokes' Theorem,

$$
\begin{aligned}
0 & =\int_{M} \operatorname{div}(\varphi \eta \nabla h) * 1 \\
& =\int_{M} \varphi \nabla \eta \cdot \nabla h * 1+\int_{M} \eta \nabla \varphi \cdot \nabla h * 1+\int_{M} \varphi \eta \Delta h * 1 \\
& =\int_{M} \varphi \nabla \eta \cdot \nabla h * 1+\int_{M} \nabla \varphi \cdot \nabla(\eta h) * 1-\int_{M} h \nabla \varphi \cdot \nabla \eta * 1+\int_{M} \varphi \eta \Delta h * 1 .
\end{aligned}
$$


Hence

$$
\int_{M} \nabla \varphi \cdot \nabla(\eta h) * 1=-\int_{M} \varphi \nabla \eta \cdot \nabla h * 1+\int_{M} h \nabla \varphi \cdot \nabla \eta * 1-\int_{M} \varphi \eta \Delta h * 1 .
$$

For arbitrary $R \leq R_{0}$, we take a cut-off function $\eta$ supported in the interior of $B_{R}, 0 \leq \eta \leq 1$, $\eta \equiv 1$ on $B_{\frac{R}{2}}$ and $|\nabla \eta| \leq c_{0} R^{-1}$. For every $\rho \leq \frac{R}{8}$, denote by $G^{\rho}$ the mollified Green function on $B_{R}$. For arbitrary $p \in B_{\frac{R}{4}}$, inserting $\varphi=G^{\rho}(\cdot, p)$ into (5.8) gives

$$
\begin{aligned}
& \int_{B_{R}} \nabla G^{\rho}(\cdot, p) \cdot \nabla(\eta h) * 1 \\
& =-\int_{B_{R}} G^{\rho}(\cdot, p) \nabla \eta \cdot \nabla h * 1+\int_{B_{R}} h \nabla G^{\rho}(\cdot, p) \cdot \nabla \eta * 1-\int_{B_{R}} G^{\rho}(\cdot, p) \eta \Delta h * 1 .
\end{aligned}
$$

We write (5.9) as

$$
I_{\rho}=I I_{\rho}+I I I_{\rho}+I V_{\rho} .
$$

By the definition of mollified Green functions,

$$
I_{\rho}=f_{B_{\rho}(p)} \eta h=f_{B_{\rho}(p)} h .
$$

Hence

$$
\lim _{\rho \rightarrow 0^{+}} I_{\rho}=h(p) .
$$

Noting that $|d \gamma|^{2}=|B|^{2}$, we have $|\nabla h| \leq\left|\nabla^{G} H\right||d \gamma|=\left|\nabla^{G} H\right||B|$. Here and in the sequel, $\nabla^{G}$ denotes the Levi-Civita connection on $\mathbf{G}_{n, m}$. In conjunction with (4.11), we have

$$
\begin{aligned}
\left|I I_{\rho}\right| & \leq \int_{T_{R}} G^{\rho}(\cdot, p)|\nabla \eta||\nabla h| * 1 \\
& \leq \sup _{T_{R}} G^{\rho}(\cdot, p) \sup _{T_{R}}|\nabla \eta| \sup _{\mathbb{V}}\left|\nabla^{G} H\right| \int_{B_{R}}|B| * 1 \\
& \leq C(n) R^{1-n} \sup _{\mathbb{V}}\left|\nabla^{G} H\right|\left(\int_{B_{R}}|B|^{2} * 1\right)^{\frac{1}{2}} \operatorname{Vol}\left(B_{R}\right)^{\frac{1}{2}} \\
& \leq c_{1}(n) \sup _{\mathbb{V}}\left|\nabla^{G} H\right|\left(R^{2-n}|B|^{2} * 1\right)^{\frac{1}{2}} .
\end{aligned}
$$

Here $T_{R} \stackrel{\text { def. }}{=} B_{R} \backslash \bar{B}_{\frac{R}{2}}$ and

$$
\mathbb{V}=\left\{P \in \mathbf{G}_{n, m}: v\left(P, P_{0}\right) \leq 3\right\},
$$

which is a compact subset of $\mathbb{U}$. 
As shown in Sect. 2, there is a one-to-one correspondence between the points in $\mathbb{U}$ and the $n \times m$-matrices. And each $n \times m$-matrix can be viewed as a corresponding vector in $\mathbb{R}^{n m}$. Define $T: \mathbb{U} \rightarrow \mathbb{R}^{n m}$

$$
Z \mapsto\left(\operatorname{det}\left(I+Z Z^{T}\right)^{\frac{1}{2}}-1\right) \frac{Z}{\left(\operatorname{tr}\left(Z Z^{T}\right)\right)^{\frac{1}{2}}}
$$

Note that $\left(\operatorname{tr}\left(Z Z^{T}\right)\right)^{\frac{1}{2}}=\left(\sum_{i, \alpha} Z_{i \alpha}^{2}\right)^{\frac{1}{2}}$ equals $|Z|$ when $Z$ is treated as a vector in $\mathbb{R}^{n m}$. Since $t \in[0,+\infty) \mapsto\left[\operatorname{det}\left(I+(t Z)(t Z)^{T}\right)\right]^{\frac{1}{2}}$ is a strictly increasing function and maps $[0,+\infty)$ onto $[1,+\infty), T$ is a diffeomorphism. By (2.6), $|T(Z)|=v\left(P, P_{0}\right)-1$. Via $T$, we can define the mean value of $\gamma$ on $B_{R}$ by

$$
\bar{\gamma}_{R}=T^{-1}\left[\frac{\int_{B_{R}}(T \circ \gamma) * 1}{\operatorname{Vol}\left(B_{R}\right)}\right] .
$$

Note that $T$ maps sublevel sets of $v\left(\cdot, P_{0}\right)$ onto Euclidean balls centered at the origin. Hence the convexity of Euclidean balls gives

$$
v\left(\bar{\gamma}_{R}, P_{0}\right) \leq \sup _{B_{R}} v\left(\cdot, P_{0}\right) \circ \gamma \leq b .
$$

The compactness of $\mathbb{V}$ ensures the existence of positive constants $K_{1}$ and $K_{2}$, such that for arbitrary $X \in T \mathbb{V}$,

$$
K_{1}|X| \leq\left|T_{*} X\right| \leq K_{2}|X| .
$$

The classical Neumann-Poincaré inequality says

$$
\int_{D_{R}}|\phi-\bar{\phi}|^{2} \leq C(n) R^{2} \int_{D_{R}}|D \phi|^{2} .
$$

As shown above, $B_{R}$ can be regarded as $D_{R}$ equipped with the metric $g=g_{i j} d x^{i} d x^{j}$, and the eigenvalues of $\left(g_{i j}\right)$ are bounded. Hence it is easy to get

$$
\int_{B_{R}}|\phi-\bar{\phi}|^{2} * 1 \leq C(n) R^{2} \int_{B_{R}}|\nabla \phi|^{2} * 1 .
$$

Here $\phi$ can be a vector-valued function.

Denote by $d_{G}$ the distance function on $\mathbf{G}_{n, m}$. Then, by using the above Neumann-Poincaré inequality we have

$$
\begin{aligned}
\int_{B_{R}} d_{G}^{2}\left(\gamma, \bar{\gamma}_{R}\right) * 1 & \leq K_{1}^{-2} \int_{B_{R}}\left|T \circ \gamma-T\left(\bar{\gamma}_{R}\right)\right|^{2} * 1 \\
& \leq C(n) K_{1}^{-2} R^{2} \int_{B_{R}}|d(T \circ \gamma)|^{2} * 1 \\
& \leq C(n) K_{1}^{-2} K_{2}^{2} R^{2} \int_{B_{R}}|d \gamma|^{2} * 1 \\
& =C(n) K_{1}^{-2} K_{2}^{2} R^{2} \int_{B_{R}}|B|^{2} * 1 .
\end{aligned}
$$


Now we write

$$
h=H \circ \gamma=H\left(\bar{\gamma}_{R}\right)+\left(H \circ \gamma-H\left(\bar{\gamma}_{R}\right)\right),
$$

then

$$
I I I_{\rho}=H\left(\bar{\gamma}_{R}\right) \int_{B_{R}} \nabla G^{\rho}(\cdot, p) \cdot \nabla \eta * 1+\int_{T_{R}}\left(H \circ \gamma-H\left(\bar{\gamma}_{R}\right)\right) \nabla G^{\rho}(\cdot, p) \cdot \nabla \eta * 1 .
$$

Similar to (5.10),

$$
\lim _{\rho \rightarrow 0^{+}} H\left(\bar{\gamma}_{R}\right) \int_{B_{R}} \nabla G^{\rho}(\cdot, p) \cdot \nabla \eta * 1=\lim _{\rho \rightarrow 0^{+}} H\left(\bar{\gamma}_{R}\right) f_{B_{\rho}(p)} \eta=H\left(\bar{\gamma}_{R}\right) .
$$

The second term can be controlled by

$$
\begin{aligned}
& \int_{T_{R}}\left(H \circ \gamma-H\left(\bar{\gamma}_{R}\right)\right) \nabla G^{\rho}(\cdot, p) \cdot \nabla \eta * 1 \\
& \leq \sup _{\mathbb{V}}\left|\nabla^{G} H\right| \sup _{T_{R}}|\nabla \eta| \int_{T_{R}} d_{G}\left(\gamma, \bar{\gamma}_{R}\right)\left|\nabla G^{\rho}(\cdot, p)\right| * 1 \\
& \leq c_{0} R^{-1} \sup _{\mathbb{V}}\left|\nabla^{G} H\right|\left(\int_{B_{R}} d_{G}^{2}\left(\gamma, \bar{\gamma}_{R}\right)\right)^{\frac{1}{2}}\left(\int_{T_{R}}\left|\nabla G^{\rho}(\cdot, p)\right|^{2} * 1\right)^{\frac{1}{2}}
\end{aligned}
$$

Substituting (4.12) and (5.15) into (5.18) yields

$$
\int_{T_{R}}\left(H \circ \gamma-H\left(\bar{\gamma}_{R}\right)\right) \nabla G^{\rho}(\cdot, p) \cdot \nabla \eta * 1 \leq c_{2}(n) \sup _{\mathbb{V}}\left|\nabla^{G} H\right|\left(R^{2-n} \int_{B_{R}}|B|^{2} * 1\right)^{\frac{1}{2}} .
$$

From (5.9), (5.11), (5.12), (5.16), (5.17) and (5.19), letting $\rho \rightarrow 0$ we arrive at

$$
\begin{aligned}
h(p) \leq & H\left(\bar{\gamma}_{R}\right)+c_{3}(n) \sup _{\mathbb{V}}\left|\nabla^{G} H\right|\left(R^{2-n} \int_{B_{R}}|B|^{2} * 1\right)^{\frac{1}{2}} \\
& -\limsup _{\rho \rightarrow 0^{+}} \int_{B_{R}} G^{\rho}(\cdot, p) \eta \Delta h * 1 .
\end{aligned}
$$

for every $p \in B_{\frac{R}{4}}$.

The compactness of $\mathbf{G}_{n, m}$ implies the existence of a positive constant $K_{3}$, such that

$$
\left|\nabla^{G} v(\cdot, P)\right| \leq K_{3} \quad \text { whenever } 1 \leq v(\cdot, P) \leq 3
$$


for arbitrary $P \in \mathbf{G}_{n, m}$. Hence by inserting $H=v(\cdot, P)$ into (5.20) one can obtain

$$
\begin{aligned}
v(\gamma(p), P) \leq & v\left(\bar{\gamma}_{R}, P\right)+c_{3} K_{3}\left(R^{2-n} \int_{B_{R}}|B|^{2} * 1\right)^{\frac{1}{2}} \\
& -\limsup _{\rho \rightarrow 0^{+}} \int_{B_{R}} G^{\rho}(\cdot, p) \eta \Delta(v(\cdot, P) \circ \gamma) * 1 .
\end{aligned}
$$

By Lemma 5.1, if we put $P_{1}=\bar{\gamma}_{R}$, then $1 \leq v\left(\cdot, P_{1}\right) \leq 3$ whenever $\left.1 \leq v\left(\cdot, P_{0}\right)\right) \leq b$ provided that $b<\frac{\sqrt{6}}{2}$, which implies $v\left(\cdot, \bar{\gamma}_{R}\right) \circ \gamma$ is a subharmonic function on $B_{R}$. Letting $P=\bar{\gamma}_{R}$ in (5.22) yields

$$
v\left(\gamma(p), \bar{\gamma}_{R}\right) \leq 1+c_{3} K_{3}\left(R^{2-n} \int_{B_{R}}|B|^{2} * 1\right)^{\frac{1}{2}}
$$

for all $p \in B_{\frac{R}{4}}$. By Theorem 4.1, for every $\varepsilon>0$, there is $\delta \in(0,1)$, depending only on $n, \beta_{0}$ and $\varepsilon$, such that

$$
R^{2-n} \int_{B_{R}}|B|^{2} * 1 \leq c_{3}^{-2} K_{3}^{-2} \varepsilon^{2}
$$

for some $R \in\left[4 \delta R_{0}, R_{0}\right]$. Substituting (5.24) into (5.23) gives (5.6).

If $b \geq \frac{\sqrt{6}}{2}$, we put $\varepsilon_{1}=\varepsilon_{1}\left(3, \beta_{0}\right)$ as given in Lemma 5.1. Then Theorem 4.1 enables us to find $R \in\left[4 \delta_{0} R_{0}, R_{0}\right]$ such that

$$
R^{2-n} \int_{B_{R}}|B|^{2} * 1 \leq \frac{1}{4} c_{3}^{-2} K_{3}^{-2} \varepsilon_{1}^{2},
$$

where $\delta_{0}$ only depends on $n$ and $\beta_{0}$. Applying Lemma 5.1, one can find $P_{1} \in \mathbf{G}_{n, m}$, such that

$$
v\left(\bar{\gamma}_{R}, P_{1}\right) \leq b-\varepsilon_{1}
$$

and $1 \leq v\left(\cdot, P_{1}\right) \leq 3$ whenever $1 \leq v\left(\cdot, P_{0}\right) \leq b$. Theorem 3.1 ensures that $v\left(\cdot, P_{1}\right) \circ \gamma$ is a subharmonic function on $B_{R}$. Taking $P=P_{1}$ in (5.22) yields

$$
v\left(\gamma(p), P_{1}\right) \leq v\left(\bar{\gamma}_{R}, P_{1}\right)+c_{3} K_{3}\left(\frac{1}{4} c_{3}^{-2} K_{3}^{-1} \varepsilon_{1}^{2}\right)^{\frac{1}{2}} \leq b-\frac{\varepsilon_{1}}{2}
$$

for all $p \in B_{\frac{R}{4}}$. Here we have used (5.25) and (5.26). From the above inequality (5.7) immediately follows.

\section{Bernstein type results}

Now we can start an iteration as in [10] and [9] to get the following estimates: 
Theorem 6.1 Let $M=\left\{(x, f(x)): x \in D_{R_{0}} \subset \mathbb{R}^{n}\right\}$ be a graph with parallel mean curvature, and $\Delta_{f} \leq \beta_{0}$ with $\beta_{0} \in[1,3)$, then for arbitrary $\varepsilon>0$, there exists $\delta \in(0,1)$, only depending on $n, \beta_{0}$ and $\varepsilon$, not depending on $f$ and $R_{0}$, such that

$$
1 \leq v(\cdot, \gamma(o)) \circ \gamma \leq 1+\varepsilon \text { on } B_{\delta R_{0}},
$$

where $o=(0, f(0))$. In particular, if $|D f|(0)=0$, then

$$
\Delta_{f} \leq 1+\varepsilon \text { on } D_{\delta R_{0}} .
$$

Proof Let $\left\{\epsilon_{1}, \ldots, \epsilon_{n+m}\right\}$ be canonical orthonormal basis of $\mathbb{R}^{n+m}$ and put $P_{0}=\epsilon_{1} \wedge \cdots \wedge \epsilon_{n}$. Then $\Delta_{f} \leq \beta_{0}$ implies $v\left(\cdot, P_{0}\right) \leq \beta_{0}$ on $B_{R_{0}}$. If $\beta_{0}<\frac{\sqrt{6}}{2}$, we put $Q_{0}=P_{0}$. Otherwise by Theorem 5.1, one can find $P_{1} \in \mathbf{G}_{n, m}$, such that

$$
v\left(\cdot, P_{1}\right) \circ \gamma \leq \beta_{0}-\varepsilon_{1} \quad \text { on } B_{\delta_{0} R_{0}}
$$

with constants $\delta_{0}$ and $\varepsilon_{1}$ depending only on $n$ and $\beta_{0}$. Similarly for each $j \geq 1$, if $\beta_{0}-j \varepsilon_{1}<$ $\frac{\sqrt{6}}{2}$, then we put $Q_{0}=P_{j}$; otherwise Theorem 5.1 enables us to find $P_{j+1} \in \mathbf{G}_{n, m}$ satisfying

$$
v\left(\cdot, P_{j+1}\right) \circ \gamma \leq \beta_{0}-(j+1) \varepsilon_{1} \quad \text { on } B_{\delta_{0}^{j+1} R_{0}} .
$$

Denoting

$$
k=\left[\left(3-\frac{\sqrt{6}}{2}\right) \varepsilon_{1}^{-1}\right]+1,
$$

then obviously $\beta_{0}-k \varepsilon_{1}<\frac{\sqrt{6}}{2}$. Hence there exists $Q_{0} \in \mathbf{G}_{n, m}$, such that

$$
v\left(\cdot, Q_{0}\right) \circ \gamma \leq b<\frac{\sqrt{6}}{2} \text { on } B_{\delta_{0}^{k} R_{0}} .
$$

Again using Theorem 5.1, for arbitrary $\varepsilon>0$, there exists $\delta_{1} \in(0,1)$, depending only on $n, \beta_{0}$ and $\varepsilon$, such that

$$
v\left(\cdot, Q_{1}\right) \circ \gamma \leq \sqrt{2}\left(1+(1+\varepsilon)^{-1}\right)^{-\frac{1}{2}} \text { on } B_{\delta_{1} \delta_{0}^{k} R_{0}}
$$

for a point $Q_{1} \in \mathbf{G}_{n, m}$. With $r(\cdot, \cdot)$ as in the proof of Lemma 5.1, then

$$
r\left(\cdot, Q_{1}\right) \circ \gamma=\arccos v\left(\cdot, Q_{1}\right)^{-1} \circ \gamma \leq \frac{1}{2} \arccos (1+\varepsilon)^{-1} .
$$

Using the triangle inequality we get

$$
r(\cdot, \gamma(0)) \circ \gamma \leq r\left(\cdot, Q_{1}\right) \circ \gamma+r\left(\gamma(0), Q_{1}\right) \circ \gamma \leq \arccos (1+\varepsilon)^{-1} .
$$

Thus $v(\cdot, \gamma(0)) \circ \gamma \leq 1+\varepsilon$ on $B_{\delta_{1} \delta_{0}^{k} R_{0}}$. It is sufficient to put $\delta=\delta_{1} \delta_{0}^{k}$.

Letting $R_{0} \rightarrow+\infty$ we can arrive at a Bernstein-type theorem:

Theorem 6.2 Let $z^{\alpha}=f^{\alpha}\left(x^{1}, \ldots, x^{n}\right), \alpha=1, \ldots, m$, be smooth functions defined everywhere in $\mathbb{R}^{n}(n \geq 3, m \geq 2)$. Suppose their graph $M=(x, f(x))$ is a submanifold with parallel mean curvature in $\mathbb{R}^{n+m}$. Suppose that there exists a number $\beta_{0}<3$ with

$$
\Delta_{f}=\left[\operatorname{det}\left(\delta_{i j}+\sum_{\alpha} \frac{\partial f^{\alpha}}{\partial x^{i}} \frac{\partial f^{\alpha}}{\partial x^{j}}\right)\right]^{\frac{1}{2}} \leq \beta_{0} .
$$

Then $f^{1}, \ldots, f^{m}$ has to be affine linear (representing an affine $n$-plane). 


\section{Final remarks}

For any $P_{0} \in \mathbf{G}_{n, m}$, denote by $r$ the distance function from $P_{0}$ in $\mathbf{G}_{n, m}$. The eigenvalues of $\operatorname{Hess}(r)$ were computed in [12]. Then define

$$
B_{J X}\left(P_{0}\right)=\left\{P \in \mathbf{G}_{n, m}: \text { sum of any two Jordan angles between } P \text { and } P_{0}<\frac{\pi}{2}\right\}
$$

in the geodesic polar coordinate neighborhood around $P_{0}$ on the Grassmann manifold. From (3.2), (3.7) and (3.9) in [12] it turns out that $\operatorname{Hess}(r)>0$ on $B_{J X}\left(P_{0}\right)$. Moreover, let $\Sigma \subset B_{J X}\left(P_{0}\right)$ be a closed subset, then $\theta_{\alpha}+\theta_{\beta} \leq \beta_{0}<\frac{\pi}{2}$ and

$$
\operatorname{Hess}(r) \geq \cot \beta_{0} g,
$$

where $g$ is the metric tensor on $\mathbf{G}_{n, m}$. Hence, the composition of the distance function with the Gauss map is a strongly subharmonic function on $M$, provided the Gauss image of the submanifold $M$ with parallel mean curvature in $\mathbb{R}^{n+m}$ is contained in $\Sigma$. The largest sub-level set of $v\left(\cdot, P_{0}\right)$ in $B_{J X}\left(P_{0}\right)$ were studied in [12]. The Theorem 3.2 in [12] shows that

$$
\max \left\{w\left(P, P_{0}\right) ; P \in \partial B_{J X}\left(P_{0}\right)\right\}=\frac{1}{2} .
$$

Therefore,

$$
\left\{P \in \mathbf{G}_{n, m}, v\left(\cdot, P_{0}\right)<2\right\} \subset B_{J X}\left(P_{0}\right),
$$

and

$$
\left\{P \in \mathbf{G}_{n, m} ; v\left(\cdot, P_{0}\right) \leq 2\right\} \bigcap \overline{B_{J X}}\left(P_{0}\right) \neq \emptyset .
$$

On the other hand, we can compute directly. From (2.7) we also have

$$
\begin{aligned}
\operatorname{Hess}\left(v\left(\cdot, P_{0}\right)\right)= & \sum_{m+1 \leq i \leq n, \alpha} v \omega_{i \alpha}^{2}+\sum_{\alpha}\left(1+2 \lambda_{\alpha}^{2}\right) v \omega_{\alpha \alpha}^{2}+\sum_{\alpha \neq \beta} \lambda_{\alpha} \lambda_{\beta} v \omega_{\alpha \alpha} \otimes \omega_{\beta \beta} \\
+\sum_{\alpha<\beta} & {\left[\left(1+\lambda_{\alpha} \lambda_{\beta}\right) v\left(\frac{\sqrt{2}}{2}\left(\omega_{\alpha \beta}+\omega_{\beta \alpha}\right)\right)^{2}\right.} \\
& \left.+\left(1-\lambda_{\alpha} \lambda_{\beta}\right) v\left(\frac{\sqrt{2}}{2}\left(\omega_{\alpha \beta}-\omega_{\beta \alpha}\right)\right)^{2}\right] .
\end{aligned}
$$

It follows that $v\left(\cdot, P_{0}\right)$ is strictly convex on $B_{J X}\left(P_{0}\right)$. Moreover, if $\theta_{\alpha}+\theta_{\beta} \leq \beta_{0}<\frac{\pi}{2}$, then

$$
\operatorname{Hess}\left(v\left(\cdot, P_{0}\right)\right) \geq\left(1-\tan \theta_{\alpha} \tan \theta_{\beta}\right) v g=\frac{\cos \left(\theta_{\alpha}+\theta_{\beta}\right)}{\cos \theta_{\alpha} \cos \theta_{\beta}} v g \geq \cos \beta_{0} v g
$$

where $g$ is the metric tensor of $\mathbf{G}_{n, m}$ and

$$
\Delta v\left(\gamma(\cdot), P_{0}\right) \geq \cos \beta_{0} v|B|^{2} \geq \cos \beta_{0}|B|^{2} .
$$

Now, we define

$$
\Sigma\left(P_{0}\right)=B_{J X}\left(P_{0}\right) \bigcup\left\{P \in \mathbf{G}_{n, m} ; v\left(\cdot, P_{0}\right)<3\right\} \subset \mathbf{G}_{n, m} .
$$

The function $v\left(\cdot, P_{0}\right)$ is not convex on whole $\Sigma\left(P_{0}\right)$. But, its composition with Gauss map could be a strongly subharmonic function on $M$.

Therefore, we could obtain a more general result: Let $M$ be a complete submanifold in $\mathbb{R}^{n+m}$ with parallel mean curvature. If its image under the Gauss map is contained in a closed subset of $\Sigma\left(P_{0}\right)$ for some $P_{0} \in \mathbf{G}_{n, m}$, then $M$ has to be an affine linear subspace. 
Acknowledgments Y. L. Xin is grateful to the Max Planck Institute for Mathematics in the Sciences in Leipzig for its hospitality and continuous support. He is also partially supported by NSFC and SFMEC.

Open Access This article is distributed under the terms of the Creative Commons Attribution License which permits any use, distribution, and reproduction in any medium, provided the original author(s) and the source are credited.

\section{References}

1. Allard, W.: On the first variation of a varifold. Ann. Math. 95, 417-491 (1972)

2. Barbosa, J.L.M.: An extrinsic rigidity theorem for minimal immersion from $S^{2}$ into $S^{n}$. J. Differ. Geom. 14(3), 355-368 (1980)

3. Chern, S.S., Osserman, R.: Complete minimal surfaces in Euclidean n-space. J. d'Anal. Math. 19, 15-34 (1967)

4. Ecker, K., Huisken, G.: A Bernstein result for minimal graphs of controlled growth. J. Differ. Geom. 31, 337-400 (1990)

5. Fischer-Colbrie, D.: Some rigidity theorems for minimal submanifolds of the sphere. Acta Math. 145, 2946 (1980)

6. Giaquinta, M., Giusti, E.: On the regularity of the minima of variational integrals. Acta Math. 148, 31$46(1982)$

7. Giaquinta, M., Hildebrandt, S.: A priori estimates for harmonic mappings. J. Reine Angew. Math. 336, 124-164 (1982)

8. Grüter, M., Widman, K.: The Green function for uniformly elliptic equations. Manuscr. Math. 37, 303342 (1982)

9. Gulliver, R., Jost, J.: Harmonic maps which solve a free-boundary problem. J. Reine Angew. Math. 381, 61-89 (1987)

10. Hildebrandt, S., Jost, J., Widman, K.: Harmonic mappings and minimal submanifolds. Invent. Math. 62, 269-298 (1980)

11. Jost, J.: Generalized Dirichlet forms and harmonic maps. Calc. Var. PDE 5, 1-19 (1997)

12. Jost, J., Xin, Y.L.: Bernstein type theorems for higher codimension. Calc. Var. 9, 277-296 (1999)

13. Jost, J., Xin, Y.L., Yang, L.: The regularity of harmonic maps into spheres and application to Bernstein problems. J. Differ. Geom. 90, 131-176 (2012)

14. Lawson, H.B., Osserman, R.: Non-existence, non-uniqueness and irregularity of solutions to the minimal surface system. Acta Math. 139, 1-17 (1977)

15. Moser, J.: On Harnack's theorem for elliptic differential equations. Commun. Pure Appl. Math. 14, 577591 (1961)

16. Ruh, E.A., Vilms, J.: The tension field of Gauss maps. Trans. AMS 149, 569-573 (1970)

17. Schoen, R., Simon, L., Yau, S.T.: Curvature estimates for minimal hypersurfaces. Acta Math. 134, 275288 (1974)

18. Wang, M.-T.: On graphic Bernstein type results in higher codimension. Trans. AMS 355(1), 265-271 (2003)

19. Wong, Y.-C.: Differential geometry of Grassmann manifolds. Proc. Natl. Acad. Sci. 57, 589-594 (1967)

20. Xin, Y.: Minimal Submanifolds and Related Topics. World Scientific Publications, Singapore (2003)

21. Xin, Y.L., Yang, L.: Convex functions on Grassmannian manifolds and Lawson-Osserman problem. Adv. Math. 219(4), 1298-1326 (2008) 